\title{
FROM CORRESPONDENCE ANALYSIS TO MULTIPLE AND JOINT CORRESPONDENCE ANALYSIS
}

\author{
Michael Greenacre \\ Departament d'Economia i Empresa \\ Universitat Pompeu Fabra \\ Ramon Trias Fargas, 25-27 \\ 08005 Barcelona. Spain \\ E-mail: michael@upf.es
}

\begin{abstract}
The generalization of simple (two-variable) correspondence analysis to more than two categorical variables, commonly referred to as multiple correspondence analysis, is neither obvious nor well-defined. We present two alternative ways of generalizing correspondence analysis, one based on the quantification of the variables and intercorrelation relationships, and the other based on the geometric ideas of simple correspondence analysis. We propose a version of multiple correspondence analysis, with adjusted principal inertias, as the method of choice for the geometric definition, since it contains simple correspondence analysis as an exact special case, which is not the situation of the standard generalizations. We also clarify the issue of supplementary point representation and the properties of joint correspondence analysis, a method that visualizes all two-way relationships between the variables. The methodology is illustrated using data on attitudes to science from the International Social Survey Program on Environment in 1993.
\end{abstract}

Keywords: Correspondence analysis, eigendecomposition, joint correspondence analysis, multivariate categorical data, questionnaire data, singular value decomposition.

Acknowledgement: The support of the BBVA Foundation (Fundación BBVA) in sponsoring this research is gratefully acknowledged. 


\section{Introduction}

Simple correspondence analysis (CA) is primarily applicable to a two-way contingency table, leading to a map that visualizes the association between two categorical variables. Multiple correspondence analysis (MCA) tackles the more general problem of associations among a set of more than two categorical variables. We shall see that the generalization to more than two variables is neither obvious nor well-defined. In other areas of multivariate analysis, such as regression and loglinear modelling, the situation is less complicated: for example, the transition from the regression of a response variable on a single predictor variable to the case of several predictors is quite straightforward. The main problem we face here is that the notion of association between two categorical variables is already a complex concept and there are several ways to generalize this concept to more than two variables.

Of the many different ways that exist to define MCA, we shall consider two approaches: first, the definition which is perhaps the easiest to understand, namely that of correlation between sets of variables, known as canonical correlation, and second, the geometric approach, which is directly linked to data visualization, and which has many similarities to Pearson-style principal component analysis. In the explanation of each approach, we will consider the case of two variables and then describe possible generalizations to more than two variables.

As an illustration of the theory, we shall use a data set from the International Social Survey Program on environment (ISSP, 1993), looking specifically at questions on attitudes towards science. The survey questions that we consider will be the following:

How much do you agree or disagree with each of these statements?

A. We believe too often in science, and not enough in feelings and faith.

B. Over all, modern science does more harm than good.

C. Any change humans cause in nature - no matter how scientific - is likely to make things worse. 
D. Modern science will solve our environmental problems with little change to our way of life.

Each question has five possible response categories: 1. agree strongly, 2. agree, 3. neither agree nor disagree, 4. disagree, 5. disagree strongly. To avoid the issue of cross-cultural differences we use data for the West German sample only (the ISSP surveys still distinguish between former West and East Germany). We shall also show how to relate the MCA results to the external demographic variables sex, age and education, also coded as categorical variables as follows:

Sex: $\quad$ male, female

Age: $\quad$ six groups, 16-24, 25-34, 35-44, 45-54, 55-64, 65 and older

Education: six groups, primary incomplete, primary completed, secondary incomplete, secondary completed, tertiary incomplete, tertiary completed

A listwise deletion of respondents with missing data has been performed, since we do not want to deal with the further complicating issue of missing data here (see Greenacre and Pardo, 2005). This reduces the original West German sample by about $14 \%$ to leave $n=$ 871 respondents with complete data, which forms the data set used in this study.

\section{Canonical correlation analysis}

\section{$2.1 \quad$ Two variables}

We start by considering just the first two variables, A (concerning belief in science) and B (concerning harm caused by science), both worded unfavourably towards science, so that disagreement indicates a favourable attitude towards science. Because there are only two variables, all 871 responses to these questions can be coded, with no loss of information, in the form of a cross-tabulation, given in Table 1. The correlational approach investigates how to measure the association between these two categorical variables. Several measures of association already exist for categorical data, some of which depend on whether the variables are measured on a nominal or ordinal scale, or whether we are trying to predict one variable from the other. In the following we shall be interested rather in the classical product-moment correlation coefficient applicable to metric data, and in the quantification of the categories, that is how to achieve numerical values for the response 
categories in order to calculate a correlation coefficient between the variables. Since the categories are ordered, a simple way out would be to use the existing values 1 to 5 , as they are coded in the data file, thereby assuming that there is an equal interval difference between adjacent points on each scale. But notice that such a choice would be incorrect if one of the variables were nominal, for example "province of residence" or "religious denomination".

There are two ways to calculate the correlation coefficient; one is from the original respondent-level data, which are the 871 pairs of responses to the two questions, or - more compactly - directly from Table 1 , since this table gives the frequencies of occurrence of all pairs of categories. Suppose that the responses to questions A and B are coded in the indicator matrices $\mathbf{Z}_{1}$ and $\mathbf{Z}_{2}$ respectively, whose columns are zero-one dummy variables: that is $\mathbf{Z}_{1}$ and $\mathbf{Z}_{2}$ are both $916 \times 5$ matrices. Then Table 1 is the cross-product $\mathbf{Z}_{1}^{\top} \mathbf{Z}_{2}$ of the two indicator matrices. Furthermore, suppose that the proposed scale values for the categories of the two variables are contained in the vectors $\mathbf{s}_{1}$ and $\mathbf{s}_{2}$, so that the individual quantified responses are in the vectors $\mathbf{Z}_{1} \mathbf{s}_{1}$ and $\mathbf{Z}_{2} \mathbf{s}_{2}$. To simplify greatly the notation, it is convenient to consider the quantified responses as initially mean-centred: $\mathbf{1}^{\top} \mathbf{Z}_{1} \mathbf{S}_{1}=\mathbf{1}^{\top} \mathbf{Z}_{2} \mathbf{S}_{2}=$ 0 , so that the covariance $s_{12}$ between the two variables and their variances $s_{1}^{2}$ and $s_{2}^{2}$ can be written as:

$$
\begin{aligned}
& s_{12}=(1 / n) \mathbf{s}_{1}^{\top} \mathbf{Z}_{1}^{\top} \mathbf{Z}_{2} \mathbf{S}_{2}=\mathbf{s}_{1}^{\top} \mathbf{P}_{12} \mathbf{s}_{2}, \\
& s_{1}^{2}=(1 / n) \mathbf{s}_{1}^{\top} \mathbf{Z}_{1}^{\top} \mathbf{Z}_{1} \mathbf{s}_{1}=\mathbf{s}_{1}^{\top} \mathbf{D}_{1} \mathbf{s}_{1} \text { and } s_{2}^{2}=(1 / n) \mathbf{s}_{2}^{\top} \mathbf{Z}_{2}^{\top} \mathbf{Z}_{2} \mathbf{s}_{2}=\mathbf{s}_{2}^{\top} \mathbf{D}_{2} \mathbf{s}_{2},
\end{aligned}
$$

where $\mathbf{P}_{12}=(1 / n) \mathbf{Z}_{1}^{\top} \mathbf{Z}_{2}$ is called the correspondence matrix, containing the relative frequencies, that is Table 1 divided by its grand total of $n=871 . \mathbf{D}_{1}$ and $\mathbf{D}_{2}$ are diagonal matrices of the marginal relative frequencies, or masses, of the two variables. Hence the correlation is equal to:

$$
r=\frac{s_{12}}{s_{1} s_{2}}=\frac{\mathbf{s}_{1}^{\top} \mathbf{P}_{12} \mathbf{s}_{2}}{\sqrt{\mathbf{s}_{1}^{\top} \mathbf{D}_{1} \mathbf{s}_{1} \mathbf{s}_{2}^{\top} \mathbf{D}_{2} \mathbf{s}_{2}}}
$$

which can be calculated directly from Table 1 and its margins. Since this calculation involves some important concepts in CA, we shall go through it in detail, using the values 1 to 5 for the categories of each variable. 
- From Table 1 we have the values of the marginal relative frequencies for the categories of the two variables:

$$
\begin{aligned}
& (1 / n) \mathbf{1}^{\top} \mathbf{Z}_{1}=(1 / 871)\left[\begin{array}{lllll}
119 & 322 & 204 & 178 & 48
\end{array}\right] \\
& =\left[\begin{array}{lllll}
0.137 & 0.370 & 0.234 & 0.204 & 0.055
\end{array}\right] \\
& (1 / n) \mathbf{1}^{\top} \mathbf{Z}_{2}=(1 / 871)\left[\begin{array}{lllll}
71 & 174 & 205 & 281 & 140
\end{array}\right]
\end{aligned}
$$

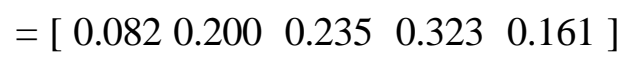

In CA these are called the masses of each of the two variables.

- Assuming the equal interval scales $1,2,3,4,5$ for the two variables, their averages are:

$$
\begin{aligned}
& (0.137 \times 1)+(0.370 \times 2)+\cdots+(0.055 \times 5)=2.672 \\
& (0.082 \times 1)+(0.200 \times 2)+\cdots+(0.161 \times 5)=3.281
\end{aligned}
$$

and the centred vectors $\mathbf{s}_{1}$ and $\mathbf{s}_{2}$ are:

$$
\mathbf{S}_{1}=\left[\begin{array}{c}
-1.672 \\
-0.672 \\
+0.328 \\
+1.328 \\
+2.328
\end{array}\right] \quad \mathbf{S}_{2}=\left[\begin{array}{l}
-2.281 \\
-1.281 \\
-0.281 \\
+0.719 \\
+1.719
\end{array}\right]
$$

- The correspondence matrix is the matrix of relative frequencies (we only give some elements of the matrix):

$$
\mathbf{P}_{12}=(1 / 871)\left[\begin{array}{ccc}
27 & \cdots & 12 \\
38 & \cdots & 30 \\
\vdots & \ddots & \vdots \\
0 & \cdots & 29
\end{array}\right]=\left[\begin{array}{ccc}
0.03100 & \cdots & 0.01378 \\
0.04363 & \cdots & 0.03444 \\
\vdots & \ddots & \vdots \\
0.00000 & \cdots & 0.03330
\end{array}\right]
$$

and the diagonal matrices of masses, $\mathbf{D}_{1}$ and $\mathbf{D}_{2}$, contain the marginal relative frequencies (masses) computed above.

- Hence, the covariance, variances and correlation are:

$$
\begin{aligned}
s_{12}=\mathbf{s}_{1}^{\top} \mathbf{P}_{12} \mathbf{S}_{2}= & (0.03100 \times-1.672 \times-2.281)+\cdots+(0.03330 \times 2.328 \times 1.719) \\
& =0.4988
\end{aligned}
$$




$$
\begin{aligned}
& s_{1}^{2}=\mathbf{s}_{1}^{\top} \mathbf{D}_{1} \mathbf{s}_{1}= 0.137 \times(-1.672)^{2}+\cdots+0.055 \times(2.328)^{2} \\
&=1.233 \\
& s_{2}^{2}=\mathbf{s}_{2}^{\top} \mathbf{D}_{2} \mathbf{s}_{2}= 0.082 \times(-2.281)^{2}+\cdots+0.161 \times(1.719)^{2} \\
&=1.412 \\
& r=\frac{s_{12}}{s_{1} s_{2}}=\frac{0.4988}{\sqrt{1.233 \times 1.412}}=0.3780
\end{aligned}
$$

All the above calculations clearly depend on the equatinterval scale values in $\mathbf{s}_{1}$ and $\mathbf{s}_{2}$ assumed at the start. We now consider these scale values as unknowns to be determined, and pose the following question: what scale values for $\mathbf{s}_{1}$ and $\mathbf{s}_{2}$ will give the highest correlation (1) between the two variables? This is exactly the problem of canonical correlation between the five dummy variables in $\mathbf{Z}_{1}$ and the five dummy variables in $\mathbf{Z}_{2}$. Since the correlation remains the same if any linear transformations of $\mathbf{s}_{1}$ and $\mathbf{s}_{2}$ are made, we need to introduce identification conditions that fix the scale of $\mathbf{a}_{1}$ and $\mathbf{a}_{2}$ in order to find the optimal solution. The usual identification conditions are that the two variables are standardized, that is that the means are zero, as before: $(1 / n) \mathbf{1}^{\top} \mathbf{Z}_{1} \mathbf{s}_{1}=(1 / n) \mathbf{1}^{\top} \mathbf{Z}_{2} \mathbf{s}_{2}=0$ and, furthermore, that the variances are 1: $\mathbf{s}_{1}^{\top} \mathbf{D}_{1} \mathbf{s}_{1}=\mathbf{s}_{2}^{\top} \mathbf{D}_{2} \mathbf{s}_{2}=1$. Under these conditions, we now show that the optimal solution coincides exactly with the so-called standard coordinates of the response categories on the first principal dimension of a simple CA of the original cross-tabulation.

Consider the singular value decomposition (SVD) of the following normalized matrix:

$$
\mathbf{D}_{1}^{-1 / 2} \mathbf{P}_{12} \mathbf{D}_{2}^{-1 / 2}=\mathbf{U S} \mathbf{V}^{\top} \quad \text { where } \mathbf{U}^{\top} \mathbf{U}=\mathbf{V}^{\top} \mathbf{V}=\mathbf{I}
$$

where $\Sigma$ is the diagonal matrix of singular values, and $\mathbf{U}$ and $\mathbf{V}$ the matrices of left and right singular vectors as columns. Then writing the equation (2) for one pair of left and right vectors, $\mathbf{u}$ and $\mathbf{v}$, corresponding to a singular value $\sigma$, we have, after multiplying on the left by $\mathbf{u}^{\top}$ and on the right by $\mathbf{v}$, and using the orthogonality of the singular vectors :

$$
\mathbf{u}^{\top} \mathbf{D}_{1}^{-1 / 2} \mathbf{P}_{12} \mathbf{D}_{2}^{-1 / 2} \mathbf{v}=\sigma
$$


So if we let $\mathbf{S}_{1}=\mathbf{D}_{1}^{-1 / 2} \mathbf{u}$ and $\mathbf{s}_{2}=\mathbf{D}_{2}^{-1 / 2} \mathbf{v}$ then $\mathbf{s}_{1}^{\top} \mathbf{P}_{12} \mathbf{S}_{2}=\sigma$, which is the formula for the covariance. Furthermore, the identification conditions $\mathbf{s}_{1}^{\top} \mathbf{D}_{1} \mathbf{s}_{1}=\mathbf{s}_{2}^{\top} \mathbf{D}_{2} \mathbf{s}_{2}=1$ are satisfied since the singular vectors have length $1: \mathbf{u}^{\top} \mathbf{u}=\mathbf{v}^{\top} \mathbf{v}=1$, so it seems that the correlation is given by the singular value $\sigma$. However, the centring conditions have not been imposed, and these can be introduced by first centring the matrix to be decomposed as follows, subtracting the product of the row and column margins from each element of the correspondence matrix:

$$
\mathbf{D}_{1}^{-1 / 2}\left(\mathbf{P}_{12}-\mathbf{P}_{12} \mathbf{1 1} \mathbf{P}_{12}^{\top}\right) \mathbf{D}_{2}^{-1 / 2}=\mathbf{U S} \mathbf{V}^{\top}
$$

where $\mathbf{P}_{12} \mathbf{1}$ is the (column) vector of row margins of $\mathbf{P}_{12}$, that is the row masses (denoted usually by $\mathbf{r}$ in simple $\mathrm{CA}$ ), and $\mathbf{1}^{\top} \mathbf{P}_{12}^{\top}$ is the (row) vector of column margins, the column masses (denoted by $\mathbf{c}$ ). In the parlance of CA this is known as "removing the trivial solution", since the uncentred matrix (2) has a trivial maximal solution with a singular value of 1 for $\mathbf{s}_{1}$ and $\mathbf{s}_{2}$ equal to $\mathbf{1}$ (thus, $\mathbf{U}, \mathbf{V}$ and $\Sigma$ in (2) all have this one extra trivial singular component which is eliminated by the centring in (3)).

We thus have the following result: each singular value is a correlation between variables $\mathrm{A}$ and $\mathrm{B}$, based on the scale values from the transformed singular vectors $\mathbf{s}_{1}$ and $\mathbf{s}_{2}$, and so the maximum correlation is attained for the first (i.e., the largest) singular value of (3), or equivalently the second largest singular value of the uncentred matrix (2). The solutions $\mathbf{s}_{1}$ and $\mathbf{s}_{2}$ are exactly the vectors of standard coordinates in CA on the first principal axis. The largest singular value $\sigma_{1}$ of (3), also called the first canonical correlation, is equal to 0.4106 in our example, compared to the value of 0.3780 obtained with the equatinterval (1-to-5) scales. The scale values are:

$$
\begin{aligned}
& \mathbf{s}_{1}^{\top}=\left[\begin{array}{lllll}
-1.017 & -0.560 & -0.248 & 1.239 & 2.741
\end{array}\right] \\
& \mathbf{s}_{2}^{\top}=\left[\begin{array}{lllll}
-1.571 & -0.667 & -0.606 & -0.293 & 1.926
\end{array}\right]
\end{aligned}
$$

These scale values are standardized, but since any linear transformation leaves the correlation unchanged, it is convenient to transform them so that the endpoints also have values 1 and 5, with a range of 4, in order to compa re with the previous equal-interval scales. For example, for the first variable, the range of values is $2.741-(-1.017)=3.758$, so 
in order to make the range exactly 4 units, we should multiply all the values by $4 / 3.758=$ 1.064 , in which case the lowest value is now $-1.017 \times 1.064=-1.083$. Then the addition of 2.083 to all the values will bring the scale to have lowest and highest values equal to 1 and 5 respectively. This procedure gives the following rescaled values for the two sets of response cate gories:

$$
\begin{aligned}
& \text { rescaled row values }=\left[\begin{array}{lllll}
1 & 1.486 & 1.818 & 3.402 & 5
\end{array}\right] \\
& \text { rescaled column values }=\left[\begin{array}{llllll}
1 & 2.034 & 2.103 & 3.132 & 5
\end{array}\right]
\end{aligned}
$$

The scale points do emerge in their expected order in both cases, but it is interesting to study their relative spacings. Compared to the equalinterval values considered previously, these rescaled values show that the categories "disagree" and "disagree strongly" for question $\mathrm{A}$ are further spaced out, with relatively small differences between scale values assigned to the categories "strongly agree", "agree" and "neither/nor". For question B the difference between "disagree" and "disagree strongly" is even larger, almost two full units. For both questions the neutral "neither/nor" category is not in the centre of the scale but close to the agreement category.

Before moving onto the case of several variables, we remark that in the above only one set of scale values has been derived for each variable, corresponding to the first singular value $\sigma_{1}$. Further sets of scale values can be determined in a stepwise manner by maximizing the correlation between another pair of subject scores based on different scale values, say $\widetilde{\mathbf{s}}_{1}$ and $\widetilde{\mathbf{s}}_{2}$, where the subject scores are uncorrelated with those already obtained., i.e. $\widetilde{\mathbf{s}}_{1}^{\top} \mathbf{D}_{1} \mathbf{s}_{1}=\widetilde{\mathbf{s}}_{2}^{\top} \mathbf{D}_{2} \mathbf{s}_{2}=0$. The solution is given by the second set of singular vectors of (3), transformed as before to standard coordinates, corresponding to the second singular value, $\sigma_{2}$, which is the second canonical correlation. For a table of order $I \times J$ this process can be continued to obtain a total of $\min \{I-1, J-1\}$ canonical correlations and associated scale values: in our $5 \times 5$ example four sets of scale values and canonical correlations can be calculated. The canonical correlations are the square roots of the principal inertias usually reported on the axes of the map (see Section 3 below). 


\subsection{Several variables}

To make the transition to the case of several variables, notice that the problem is almost identical if we reformulate it as maximizing the correlation between the two variables and their average (or their sum). In general, for two variables $z_{1}$ and $z_{2}$ with correlation $\rho$, the correlation between either of them and their average $1 / 2\left(z_{1}+z_{2}\right)$ (or their sum $\left.z_{1}+z_{2}\right)$ is equal to $\sqrt{(1+?) / 2}$, so that maximizing $\rho$ is equivalent to maximizing the correlation between the variables and their average (or sum). The only real difference is the value of the maximum found: in the latter formulation this will be $\sqrt{(1+?) / 2}$ and not the value of $\rho$ itself. The average of two categorical variables leads us to consider the matrix of the two indicator matrices $\left[\begin{array}{ll}\mathbf{Z}_{1} & \mathbf{Z}_{2}\end{array}\right]$, where the average of the two quantifications of the variables, based on $\mathbf{s}_{1}$ and $\mathbf{s}_{2}$ respectively, is equal to

$$
\frac{1}{2}\left(\mathbf{Z}_{1} \mathbf{s}_{1}+\mathbf{Z}_{2} \mathbf{s}_{2}\right)=\frac{1}{2}\left[\begin{array}{ll}
\mathbf{Z}_{1} & \mathbf{Z}_{2}
\end{array}\right]\left[\begin{array}{l}
\mathbf{s}_{1} \\
\mathbf{s}_{2}
\end{array}\right]
$$

Consider now what happens when we apply the standard CA algorithm to the superindicator matrix $\mathbf{Z}=\left[\begin{array}{ll}\mathbf{Z}_{1} & \mathbf{Z}_{2}\end{array}\right]$. Since $\mathbf{Z}$ has total sum $2 n$, with each of the $n$ rows summing to a constant 2 and column sums equal to the marginal frequencies of each variable, the correspondence matrix is $(1 / 2 n) \mathbf{Z}$, the row mass matrix is $(1 / n) \mathbf{I}$ and the column mass matrix is $\mathbf{D}=1 / 2 \operatorname{diag}\left(\mathbf{D}_{1}, \mathbf{D}_{2}\right)$, where $\operatorname{diag}\left(\mathbf{D}_{1}, \mathbf{D}_{2}\right)$ is the diagonal matrix formed by the two diagonal matrices $\mathbf{D}_{1}$ and $\mathbf{D}_{2}$ defined. Hence the SVD to compute the CA solution of $\mathbf{Z}$ is (in its uncentred form - cf. (2)):

$$
\sqrt{n} \frac{\mathbf{Z}}{2 n} \mathbf{D}^{-1 / 2}=\mathbf{U G V}^{\top} \quad \text { where } \mathbf{U}^{\top} \mathbf{U}=\mathbf{V}^{\top} \mathbf{V}=\mathbf{I}
$$

which - in one of its symmetric eigenvalue formulations - can be written as:

$$
\left(\sqrt{n} \frac{\mathbf{Z}}{2 n} \mathbf{D}^{-1 / 2}\right)^{\top}\left(\sqrt{n} \frac{\mathbf{Z}}{2 n} \mathbf{D}^{-1 / 2}\right)=\frac{1}{4 n} \mathbf{D}^{-1 / 2} \mathbf{Z}^{\top} \mathbf{Z D}^{-1 / 2}=\mathbf{V G}^{2} \mathbf{V}^{\top} \quad \text { where } \mathbf{V}^{\top} \mathbf{V}=\mathbf{I}
$$

that is

$$
\frac{1}{4 n} \mathbf{D}^{-1 / 2} \mathbf{C D}^{-1 / 2}=\mathbf{V G}^{2} \mathbf{V}^{\top}=\mathbf{V} ? \mathbf{V}^{\top}
$$


where $\mathbf{C}=\mathbf{Z}^{\top} \mathbf{Z}$ and $\Lambda=\Gamma^{2}$. The matrix $\mathbf{C}$, called the Burt matrix, is an important data structure in MCA: it is the matrix of all two-way cross-tabulations of the categorical variables, which in this case two categorical variables can be written:

$$
\mathbf{C}=\left[\begin{array}{ll}
\mathbf{Z}_{1}^{\top} \mathbf{Z}_{1} & \mathbf{Z}_{1}^{\top} \mathbf{Z}_{2} \\
\mathbf{Z}_{2}^{\top} \mathbf{Z}_{1} & \mathbf{Z}_{2}^{\top} \mathbf{Z}_{2}
\end{array}\right]=n\left[\begin{array}{ll}
\mathbf{D}_{1} & \mathbf{P}_{12} \\
\mathbf{P}_{21} & \mathbf{D}_{2}
\end{array}\right]
$$

We use the notation $\Lambda=\Gamma^{2}$, that is the squares $\gamma^{2}$ of the singular values, or principal inertias of $\mathbf{Z}$ that appear on the diagonal of $\Gamma^{2}$, are denoted by $\lambda$, on the diagonal of $\Lambda$. Writing (4) for a single eigenvector $\mathbf{v}$, partitioned into two subvectors $\mathbf{v}_{1}$ and $\mathbf{v}_{2}$ (one corresponding to the rows of the original table, the other to the columns) and multiplying as before on the left by $\mathbf{v}^{\top}$ and on the right by $\mathbf{v}$, defining $\mathbf{S}=\mathbf{D}^{-1 / 2} \mathbf{v}$ similarly partitioned into $\mathbf{s}_{1}$ and $\mathbf{s}_{2}$, we obtain the eigenequation:

$$
\frac{1}{4}\left[\begin{array}{l}
\mathbf{s}_{1} \\
\mathbf{s}_{2}
\end{array}\right]^{\top}\left[\begin{array}{ll}
\mathbf{D}_{1} & \mathbf{P}_{12} \\
\mathbf{P}_{21} & \mathbf{D}_{2}
\end{array}\right]\left[\begin{array}{l}
\mathbf{s}_{1} \\
\mathbf{s}_{2}
\end{array}\right]=\boldsymbol{\gamma}^{2}=\boldsymbol{\lambda}
$$

that is:

$$
{ }_{1 / 4}\left(\mathbf{S}_{1}^{\top} \mathbf{D}_{1} \mathbf{S}_{1}+\mathbf{S}_{1}{ }^{\top} \mathbf{P}_{12} \mathbf{S}_{2}+\mathbf{S}_{2}{ }^{\top} \mathbf{P}_{21} \mathbf{S}_{1}+\mathbf{S}_{2}^{\top} \mathbf{D}_{2} \mathbf{S}_{2}\right)=\gamma^{2}=\lambda
$$

The maximum value of (5), given by the largest nontrivial eigenvalue $\lambda_{1}=\gamma_{1}^{2}$, coincides with the solution of simple CA of the single two-way table with correspondence matrix $\mathbf{P}_{12}$, except that its maximum is now equal to $1 / 4\left(1+\sigma_{1}+\sigma_{1}+1\right)=1 / 2\left(1+\sigma_{1}\right)$, where $\sigma_{1}$ is the maximized canonical correlation in simple CA. According to our previous remarks $\lambda_{1}=1 / 2\left(1+\sigma_{1}\right)$ is exactly the square of the correlation between either of the two (quantified) categorical variables and their average (or sum). Hence $\mathbf{s}_{1}$ and $\mathbf{s}_{2}$ derived above are identical to the $\mathbf{s}_{1}$ and $\mathbf{s}_{2}$ of simple $\mathrm{CA}$, and what we have derived are the standard coordinates of the columns of the indicator matrix $\mathbf{Z}$. Notice that the eigenvalue $\lambda_{1}$ above is also the singular value of $\mathbf{C}$, since $\mathbf{C}$ is symmetric: in the language of geometric CA (see Section 3 below) $\lambda_{1}$ is the square root of the principal inertia of the Burt matrix $\mathbf{C}$.

The alert reader will have noticed that in (4) the identification condition on $\mathbf{s}$ implied by the standardization of $\mathbf{v}$ in the SVD is that its weighted sum of squares is equal to 1 , that is $1 / 2\left(\mathbf{s}_{1}^{\top} \mathbf{D}_{1} \mathbf{s}_{1}+\mathbf{s}_{2}^{\top} \mathbf{D}_{2} \mathbf{s}_{2}\right)=\mathbf{s}^{\top} \mathbf{D s}=1$, and not that the subvectors $\mathbf{s}_{1}$ and $\mathbf{s}_{2}$ are 
individually normalized to be 1 . It can be shown, however, that if $\mathbf{s}_{1}$ and $\mathbf{s}_{2}$ constitute a solution corresponding to an eigenvalue $1 / 2(1+\sigma)$, then $\mathbf{s}_{1}$ and $-\mathbf{s}_{2}$ constitute another solution corresponding to the eigenvalue $1 / 2(1-\sigma)$ (see, for example, Greenacre, 1984: Section 5.1). The orthogonality of these eigenvectors, $\mathbf{s}_{1}{ }^{\top} \mathbf{D}_{1} \mathbf{s}_{1}-\mathbf{s}_{2}^{\top} \mathbf{D}_{2} \mathbf{s}_{2}=0$, together with the overall normalization constraint, imply the individual normalizations $\mathbf{s}_{1}{ }^{\top} \mathbf{D}_{1} \mathbf{s}_{1}=\mathbf{s}_{2}^{\top} \mathbf{D}_{2} \mathbf{s}_{2}=$ 1. As far as the individual centring constraints are concerned, these are automatic since each set of dummy variables (columns of $\mathbf{Z}_{1}$ and $\mathbf{Z}_{2}$ ) has the same sum, equal to $\mathbf{1}$, the vector of ones, so that each set has the same centroid, equal to the overall centroid $(1 / n) \mathbf{1}$.

The scene is now set for one possible generalization of CA to the multivariable case, where there are $Q$ categorical variables, coded in indicator matrices $\mathbf{Z}_{1}, \mathbf{Z}_{2}, \ldots, \mathbf{Z}_{Q}$. The problem can be defined as finding a set of scale values $\mathbf{s}_{1}, \mathbf{s}_{2}, \ldots, \mathbf{s}_{Q}$ for the variables so that an overall measure of correlation is maximized. To generalize the two-variable case, the measure of choice is the sum of squared correlations of the individual scores $\mathbf{Z}_{1} \mathbf{s}_{1}, \mathbf{Z}_{2} \mathbf{s}_{2}, \ldots$, $\mathbf{Z}_{Q} \mathbf{s}_{Q}$ with the summated score $\mathbf{Z} \mathbf{s}$, where $\mathbf{Z}$ and $\mathbf{s}$ are the concatenations of the $\mathbf{Z}_{q}$ 's and $\mathbf{s}_{q}{ }^{\text {'s }}$ respectively. We specify an overall identification constraint $\mathbf{s}^{\top} \mathbf{D} \mathbf{s}=1$, where $\mathbf{D}=$ $(1 / Q) \operatorname{diag}\left(\mathbf{D}_{1}, \mathbf{D}_{2}, \ldots, \quad \mathbf{D}_{Q}\right)$. This overall constraint does not imply that individual variances $\mathbf{s}_{q}^{\top} \mathbf{D}_{q} \mathbf{s}_{q}$ will be 1 in the final solution - in contrast to the case $Q=2$ described in the previous paragraph.

Again there are two ways to achieve the solution, one way by performing a CA of the super-indicator matrix $\mathbf{Z}=\left[\begin{array}{llll}\mathbf{Z}_{1} & \mathbf{Z}_{2} & \ldots \mathbf{Z}_{Q}\end{array}\right]$, alternatively a $\mathrm{CA}$ of the Burt matrix $\mathbf{C}$, which is now a block matrix with $Q$ blocks row-wise and column-wise. We denote the number of categories for the $q$-th categorical variable by $J_{q}$, and let $J=\Sigma_{q} J_{q}$ be the total number of categories. Then $\mathbf{Z}$ is of order $n \times J$ and $\mathbf{C}$ is of order $J \times J$. Since $\mathbf{Z}$ has total sum $n Q$, with row sums equal to a constant $Q$ and column sums equal to the marginal frequencies of each variable, the correspondence matrix is $(1 / Q n) \mathbf{Z}$, the row mass matrix is $(1 / n) \mathbf{I}$ and the column mass matrix is $\mathbf{D}$. Hence the SVD to compute the CA solution of $\mathbf{Z}$ is (in its uncentred form - see (2)):

$$
\sqrt{n} \frac{\mathbf{Z}}{Q n} \mathbf{D}^{-1 / 2}=\mathbf{U G V} \mathbf{T}^{\top} \quad \text { where } \mathbf{U}^{\top} \mathbf{U}=\mathbf{V}^{\top} \mathbf{V}=\mathbf{I}
$$


To eliminate the trivial solution the matrix to be decomposed is (see (3)): $\sqrt{n}\left(\frac{\mathbf{Z}}{Q n}-\frac{1}{n} \mathbf{1 1}^{\top} \mathbf{D}\right) \mathbf{D}^{-1 / 2}$ where $(1 / n) \mathbf{1}$ is the vector of row masses and $\mathbf{1}^{\top} \mathbf{D}$ is the vector of column masses of the indicator matrix (denoted by $\mathbf{c}^{\top}$ in simple CA). The SVD for the CA of the Burt matrix $\mathbf{C}$ (uncentred) is:

$$
\mathbf{D}^{-1 / 2} \frac{\mathbf{C}}{Q^{2} n} \mathbf{D}^{-1 / 2}=\mathbf{V} \mathbf{G}^{2} \mathbf{V}^{\top}=\mathbf{V} ? \mathbf{V}^{\top} \text { where } \mathbf{V}^{\top} \mathbf{V}=\mathbf{I}
$$

and $\mathbf{C}=\mathbf{Z}^{\top} \mathbf{Z}$. Once again, the centred form of the matrix on the left hand side of (7) removes the trivial solution in the form of the expected relative frequencies: $\mathbf{D}^{-1 / 2}\left(\frac{\mathbf{C}}{Q^{2} n}-\mathbf{D 1 1}^{\top} \mathbf{D}\right) \mathbf{D}^{-1 / 2}$.

The right singular vectors, which give us the scale values for the $Q$ variables, are identical in the two problems. The maximum value of the average squared correlation is given by the square of the first singular value in the (centred) analysis of $\mathbf{Z}$, that is the first singular value in the (centred) analysis of $\mathbf{C}$. Notice that, as in the two-variable case, the singular values in the analysis of $\mathbf{C}$ are also eigenvalues, since the matrix being decomposed is positive definite symmetric. The standard coordinates $\mathbf{x}$ which provide the scale values, partitioned into $\mathbf{x}_{1}, \mathbf{x}_{2}, \ldots, \mathbf{x}_{Q}$ for the $Q$ variables, are given by the usual transformation of the singular vectors: $\mathbf{x}=\mathbf{D}^{-1 / 2} \mathbf{v}$ where $\mathbf{v}$ is the first right singular vector, that is the first column of $\mathbf{V}$. Only the principal coordinates are slightly different in the two problems, since the singular values differ.

We now apply the above theory to the four variables described in Section 2.1. In terms of our notation: $Q=4, J_{q}=5$ for all $q, J=20, \mathbf{Z}$ is $871 \times 20$ and $\mathbf{C}$ is $20 \times 20$. The full Burt matrix is reproduced in Table 2. In Table 3 we reproduce the standard coordinates for the first and second optimal solutions, along with their corresponding correlation measures. In addition, the squared correlation of each quantified variable with the total score is given, showing that the correlation measure is equal to their average. The first and optimal set of scale values, with an average squared correlation of 0.457 , are monotonically increasing for questions $\mathrm{A}, \mathrm{B}$ and $\mathrm{C}$, but question $\mathrm{D}$ has a quite different pattern, with the extreme poles opposing the intermediate categories. This is evidence that 
there is a possible problem with the responses to question $\mathrm{D}$, which was worded in the reverse sense compared to the other questions. The second set of scale values captures an axis of "polarisation" where all four questions have the pattern of the extreme categories opposing the intermediate ones, and here question D fits in more with the others. This interpretation is supported by the squared correlations, which show a low value for question $\mathrm{D}$ in the first solution. MCA thus effectively acts as an item analysis and this results shows us that question D has degraded the reliability of the total score based on the second optimal solution, and should preferably be removed.

To clarify the link between MCA and reliability theory, consider the $Q$ variables as items measuring an underlying construct. Using the average squared correlation of 0.457 , that is 0.676 in the square root, as a measure of the reliability is an overestimate, because even for random data we would find positive correlation between items and their sum (in fact the average squared correlation between $\mathrm{Q}$ uncorrelated items and their sum is equal to $1 / Q)$. Cronbach's alpha is a measure of reliability that compensates for this and is classically defined as:

$$
\alpha=\left(\frac{Q}{Q-1}\right)\left(1-\frac{\sum_{q} s_{q}^{2}}{s^{2}}\right)
$$

where $s_{q}{ }^{2}$ is the variance of the $q$-th item score and $s^{2}$ is the variance of the summated score. In MCA the sum of item score variances $\left(\Sigma_{q} s_{q}^{2}\right)$ is equal to $\mathbf{a}^{\top} \mathbf{D a}$, which from the identification conditions described above is a fixed value, equal to $Q$. The variance of the summated score $\left(s^{2}\right)$ is equal to $Q^{2}$ times the variance of the average score $\mathbf{z}$, that is $Q^{2}$ times the $\lambda$ that we are maximizing. Hence, we can write the maximum value of (8) as:

$$
\alpha=\left(\frac{Q}{Q-1}\right)\left(1-\frac{Q}{Q^{2} \lambda}\right)=\left(\frac{Q}{Q-1}\right)\left(1-\frac{1}{Q \lambda}\right)
$$

so that maximum $\lambda$ (the first singular value of $\mathbf{C}$ in (7), which is also an eigenvalue as we have said before) corresponds to maximum reliability. Hence, the maximum value of Cronbach's $\alpha$ for the first two solutions is, respectively (see Table 3): 


$$
\alpha_{1}=\frac{4}{3}\left(1-\frac{1}{4 \times 0.457}\right)=0.605 \quad \alpha_{2}=\frac{4}{3}\left(1-\frac{1}{4 \times 0.431}\right)=0.560
$$

If question $\mathrm{D}$ is removed, as would be suggested by its low item correlation with the total, a recomputation of the solution gives a much higher value, 0.602 , of the maximum average squared correlation, and an increase in Cronbach's alpha to 0.669 (we do not report the complete results here).

Table 4 shows all the squared intercorrelations as well as the variances and covariances of the four quantified questions, according to the first optimal solution. This table also demonstrates empirically that the optimal $\lambda$ can be computed either as (i) the variance of the total score, or (ii) the average of the four squared correlations of the respective questions with the total, or (iii) the average of all the elements of the full variance-covariance matrix between the four questions.

\subsection{Homogeneity analysis}

An alternative but equivalent definition of the correlational definition of MCA is based on Guttman's criterion of "internal consistency" (see, for example, Nishisato, 1994). The idea is to look for scale values $\mathbf{s}_{1}, \mathbf{s}_{2}, \ldots, \mathbf{s}_{Q}$ which give individual scores $\mathbf{Z}_{1} \mathbf{s}_{1}, \mathbf{Z}_{2} \mathbf{s}_{2}$, $\ldots, \mathbf{Z}_{Q} \mathbf{s}_{Q}$ that are as close to one another (i.e., homogeneous) as possible. Lack of closeness can be measured by the sum of squares of the differences of each individual's $Q$ scale values from the corresponding mean score in the vector $(1 / Q)\left(\mathbf{Z}_{1} \mathbf{s}_{1}+\mathbf{Z}_{2} \mathbf{s}_{2}+\ldots+\mathbf{Z}_{Q} \mathbf{s}_{Q}\right)=$ $(1 / Q) \mathbf{Z s}$, which we shall again denote by $\mathbf{z}$. The overall objective is thus to minimize in this case the following function of $\mathbf{s}$, which is the average of the $Q$ squared differences for each individual, averaged in turn over all $n$ individuals:

$$
\frac{1}{n Q}\left[\left(\mathbf{Z}_{1} \mathbf{s}_{1}-\mathbf{z}\right)^{\top}\left(\mathbf{Z}_{1} \mathbf{s}_{1}-\mathbf{z}\right)+\left(\mathbf{Z}_{2} \mathbf{s}_{2}-\mathbf{z}\right)^{\top}\left(\mathbf{Z}_{2} \mathbf{s}_{2}-\mathbf{z}\right)+\cdots+\left(\mathbf{Z}_{Q} \mathbf{s}_{Q}-\mathbf{z}\right)^{\top}\left(\mathbf{Z}_{Q} \mathbf{s}_{Q}-\mathbf{z}\right)\right]
$$

This approach is known as homogeneity analysis (Gifi, 1990) and the objective function (10) is called the loss function. Here "loss" refers to loss of homogeneity, since perfect homogeneity would be when all the differences $\mathbf{Z}_{q} \mathbf{s}_{q}-\mathbf{z}$ are zero. Once more an identification condition on $\mathbf{s}$ is required, otherwise the trivial solution when all elements of $\mathbf{s}$ are constant will be found, giving a loss of 0 . With the same quadratic constraint $\mathbf{s}^{\top} \mathbf{D s}=1$ 
as before, it can be shown that minimum loss is achieved by the same optimal scale values described above, and the value of the minimum is equal to 1 minus the value of the corresponding largest eigenvalue of the super-indicator matrix $\mathbf{Z}$. In our example, the successively maximized eigenvalues of 0.457 and 0.431 (see Table 4) correspond to minimum losses of 0.543 and 0.569 respectively.

\section{Geometric approach}

The geometric approach to CA (see, for example, Greenacre, 1993a), turns out to be slightly more problematic to generalize to the multivariable case. A lot of the controversy about CA stems from this difficulty, and here we shall clarify the issues involved in MCA as a graphical method as well as propose a specific version of MCA that acceptably addresses these problems. We shall approach the geometry from both the chi-square distance scaling perspective and the biplot perspective.

Figure 1 shows the usual CA map of the contingency table in Table 1. The map is established using the theory described in Section 2.1, namely the SVD of the matrix of standardized residuals, followed by the calculation of the principal coordinates to represent the points in a map. The principal coordinates are the standard coordinates multiplied by the respective singular values. Since standard coordinates have unit normalization, principal coordinates are normalized to have (weighted) sum of squares equal to the respective squared singular value of the associated solution. The squared singular value $\sigma^{2}$ is called the principal inertia, corresponding to a principal axis, or dimension. Figure 1 shows the points represented by their principal coordinates calculated for the first two principal axes.

\subsection{Chi-square distance scaling}

Simple CA is justified mainly by its use of the chi-square $\left(\chi^{2}\right)$ distance as a measure of dissimilarity between row profiles and between column profiles of a two-way table. In Figure 1, where both rows and columns are displayed in principal coordinates, distances between row points optimally approximate the $\chi^{2}$ distances between row profiles and the distances between column points optimally approximate the $\chi^{2}$ distances between column 
profiles. Recall that the squared $\chi^{2}$ distance between the row profiles, for example, has this form:

$$
d^{2}\left(i, i^{\prime}\right)=\sum_{j=1}^{J}\left(\frac{p_{i j}}{r_{i}}-\frac{p_{i^{\prime} j}}{r_{i^{\prime}}}\right)^{2} / c_{j}
$$

so that every $j$-th squared difference between the profile elements is weighted inversely by the column margin $c_{j}$.

MCA is the application of CA to either the super-indicator matrix $\mathbf{Z}$ or the Burt matrix C. While the $\chi^{2}$ distance makes sense for a two-way contingency table, it has less justification when applied to the rows and to the columns of the super-indicator matrix or the Burt matrix. As an illustration of this problem consider the same four-variable example on attitudes to science in the environmental context. As shown in (11) the $\chi^{2}$ distances between rows and between columns are calculated between their profiles - in the case of distances between row profiles the column masses of the correspondence matrix are used inversely as weights in the calculation of distance. The row profiles of the super-indicator matrix $\mathbf{Z}=\left[\begin{array}{llll}\mathbf{Z}_{1} & \mathbf{Z}_{2} & \mathbf{Z}_{3} & \mathbf{Z}_{4}\end{array}\right]$ are vectors with elements equal to 0 apart from four values of $1 / 4$ in the positions of the categories selected by the corresponding case. When calculating the distance between two rows, differences between coincident zero values and coincident values of $1 / 4$ are zero, thus making no contribution to the distance measure, and so it is only differences between non-coincident categories that count in the distance function. These nonzero squared differences (each equal to $1 / 16$ in this case), arising from disagreements between respondents, are then weighted by the inverses of the corresponding column masses, proportional to the respective categories' marginal frequencies, and added up to give the $\chi^{2}$ distances. For the rows this distance measure appears fairly reasonable, and the weighting is in accordance with the $\chi^{2}$ concept that the contribution of categories with low frequency needs to be boosted since their variance is inherently lower.

The situation for the column profiles of $\mathbf{Z}$, however, is quite different and difficult, if not impossible, to justify. Here we make the distinction between calculating (i) distances between two categories of the same variable and (ii) distances between two categories of different variables. Let us denote the relative frequency of the $j$-th column category by $c_{j}$ 
(that is, for a particular variable, the quantities $c_{j}$ sum to 1). As shown by Greenacre (1989), the squared $\chi^{2}$ distances between two column categories of a super-indicator matrix is in the two cases:

i. $1 / c_{j}+1 / c_{j^{\prime}}$ between categories $j$ and $j^{\prime}$ of the same variable $q$

ii. $1 / c_{j}+1 / c_{j^{\prime}}-2 p_{j j^{\prime}} /\left(c_{j} c_{j^{\prime}}\right)$ between categories $j$ and $j^{\prime}$ of different variables $q$ and $q^{\prime}$ where $p_{i j^{\prime}}$ is the relative frequency of occurrence of categories $j$ and $j^{\prime}$ (in fact the above formulas are the same, since the frequency of co-occurrence of categories $j$ and $j^{\prime}$ of the same variable is zero). The former "within-variable" distance makes little sense since it depends only on the marginal frequencies, no matter what the relationship with the other variables is. The latter "between-variable" distance has at least a slight justification in that the distance decreases as association between categories $j$ and $j^{\prime}$ increases, but again the dominant role played by the marginal frequencies is hard to defend.

The situation improves if we consider inter-category distances calculated on the Burt matrix rather than the indicator matrix. Since the Burt matrix is symmetric it makes no difference whether we calculate the $\chi^{2}$ distances between rows or between columns. The squared distance between categories can be described verbally as follows:

i. between categories $j$ and $j$ ' of the same variable $q$ : this within-variable squared distance is the average of the $(Q-1)$ squared $\chi^{2}$ distances between categories $j$ and $j^{\prime}$ calculated in the cross-tabulations of variable $q$ with all the other variables $q^{\prime} \neq q$, but also including an unnecessary term from the cross-tabulation of $q$ with itself (this term involves the distance between two unit profiles in a submatrix on the diagonal of $\mathbf{C}$ and is thus a large component of the overall distance, tending to inflate the distance);

ii. between categories $j$ and $j^{\prime}$ of different variables $q$ and $q^{\prime}$ : this between-variable squared distance is an average of $(Q-2)$ squared $\chi^{2}$ distances between profiles of categories $j$ and $j^{\prime}$ across variables not equal to $q$ or $q^{\prime}$, but also including two additional terms which can also be considered unnecessary (these measure distances between a profile and a unit profile again on the diagonal of $\mathbf{C}$, again tending to inflate the between-category distance). 
In spite of the above theoretical difficulties to justify the full-space chi-square geometry, MCA as regularly applied - that is, the CA of $\mathbf{Z}$ or of $\mathbf{C}$ - successfully recovers interesting patterns of association between the variables. It seems that the low-dimensional projections of the points are more valid than their full-dimensional counterparts, which is a paradox from the multidimensional scaling viewpoint. Another worrying aspect is the inflation of the total inertias of $\mathbf{Z}$ and of $\mathbf{C}$, which leads to all percentages of inertia on the principal axes being artificially low. This inflation can also be understood by considering the calculation of total inertia for the Burt matrix $\mathbf{C}$ and the high contributions made by the diagonal matrices on its block diagonal. It is clear that MCA of a two-variable data set will not give the same results a a CA - the standard coordinates will be the same, but the principal inertias (and hence the principal coordinates) and their percentages of inertia will be different. In Section 3.3 below we define another variant of MCA, called joint correspondence analysis, which resolves all these issues to a certain extent. We shall also show that a simple adjustment of the scale in the MCA solution dramatically improves the fit from a multidimensional scaling viewpoint.

\subsection{Biplot}

The biplot is concerned with data reconstruction in a joint map of the rows and columns, rather than distance reconstruction. In the simple case of a twoway table one can think of reconstructing different variants of the table depending on the way we think of the table: either as a set of rows, or a set of columns, or just a two-way table of entries where rows and columns are symmetric entities. As an illustration of this approach we consider a twoway table as a set of rows, for example Table 5(a) shows the row profiles of the two-way table in Table 1, that is conditional on each response category of question $A$, the proportions of respondents falling into the response categories of question B. The biplot can be thought of as a way to reconstruct these row profiles in a map. Greenacre and Hastie (1987) and (1993a, 1993b) show how the asymmetric map of CA, with row points in principal coordinates and column points in standard coordinates, is a biplot of these profiles. The direction vector defined by each column point, called a biplot axis, can be calibrated in profile units, and the approximate value of the profile can be read off the map by simply projecting the row points onto the column axis (Figure 2). The success of the 
reconstruction of the data from the biplot in this way is measured by the percentage of inertia explained by the map: in this case it is $95.6 \%$, so the reconstruction has an error of only $4.4 \%$. Table 5(b) reports the estimated values from the biplot of Figure 2, testifying to the high accuracy of the data reconstruction.

Interpreting Figure 2 we can see, for example, a direction opposing the categories B2 and B3 pointing bottom left and category B5 top right. If we project A1, A2 and A3 onto this diagonal "dimension", it is clear that they project more or less at the same position, showing that their profile values on B2, B3 and B5 are similar, with profile values on B2 and B3 above average, and those on B5 below average (the origin of the biplot always represents the average profile values exactly). This deduction from the map can be confirmed in Table 5(a), and it is only for categories A4 and A5 that there are distinct changes along this direction, increasing in percentage response to B5 and decreasing on B2 and B3. Likewise, with respect to the other diagonal "dimension" from top left to bottom right, which opposes B1 and B4, we see that A3, A4 and A5 project at the same positions and thus are estimated to have similar profile values on B1 and B4. This can be mostly verified in Table 5(a), the only exception being the profile of A5 on B4, which has an observed frequency much lower than the corresponding values for A3 and A4 - this error of approximation would be part of the $4.4 \%$ unexplained inertia.

Thinking about the joint map in this way is sheds light on the problematic aspects of the $\mathbf{C A}$ of the indicator matrix $\mathbf{Z}$ or the Burt matrix $\mathbf{C}$. In the case of $\mathbf{Z}$ it is futile to expect a good approximation of a matrix of zeros and ones in a two-dimensional map of points. Another measure of quality is needed, for example one could deduce from a joint map the most likely set of responses of each case (row) and then count how many of these are correct predictions (see Gower, 1993; Gower and Harding, 1998; Greenacre, 1998). The situation is similar for the Burt matrix $\mathbf{C}$ : any attempt to approximate the diagonal matrices down the diagonal of the Burt matrix is clearly in conflict with the approximation of the more interesting and relevant contingency tables in the rest of the matrix. In both cases the percentages of inertia will be artificially low because of the structurally high-dimensional nature of the matrices being analyzed. 
Figure 3 shows the CA of the Burt matrix of Table 3, and notice that it only represents $35.1 \%$ of the total inertia. Yet its interpretation is clear: we can see the same pattern of association for questions A and B already seen in Figure 1, along with a similar pattern of association with question C. But the response categories for question D are not at all in line with the other three. The categories D1 and D5 of strong agreement and strong disagreement lie within the arch formed by the other questions, quite close together even though they are at opposite ends of the scale. This shows clearly the incompatibility of this question with the others.

Notice how different the scale of Figure 3 is compared to Figure 1, and how the points have been pulled outwards in the analysis of the Burt matrix. Most of the problem of low percentages of inertia is due to this scale change, and can be rectified by a simple scale adjustment of the solution. This is best explained after an account of joint correspondence analysis.

\subsection{Joint correspondence analysis}

As we have just seen, when applying CA to the Burt matrix the diagonal submatrices on the "diagonal" of the block matrix $\mathbf{C}$ inflate both the chi-square distances between profiles and the total inertia by artificial amounts. In an attempt to generalize simple CA more naturally to more than two categorical variables, joint correspondence analysis (JCA) accounts for the variation in the "off-diagonal" tables of $\mathbf{C}$ only, ignoring the matrices on the block diagonal. Hence, in the two variable case $(Q=2)$ when there is only one off-diagonal table, JCA is identical in all respects to simple CA (which is not the case for MCA of $\mathbf{Z}$ or C, which give different principal inertias).

The solution can no longer be obtained by a single application of the SVD and various algorithms have been proposed by Greenacre (1988), Boik (1996) and Tateneni and Browne (2000). For example, Greenacre (1988) describes an alternating least-squares algorithm that treats the matrices on the block diagonal as missing values. The algorithm proceeds in the following steps:

1. Perform MCA by applying CA to the Burt matrix $\mathbf{C}$, and choose the dimensionality $S^{*}$ of the solution (e.g., $S^{*}=2$ is the most typical). 
2. Optionally perform an adjustment of the solution along each of the $S^{*}$ dimensions to improve the approximation to the off-diagonal block matrices (see Section 3.4 below).

3. From the resulting map, reconstruct the values in the diagonal blocks of $\mathbf{C}$ in the same way as the data were reconstructed in the biplot, using the reconstruction formula. Replace the original values in the diagonal blocks by these estimates, calling this the modified Burt matrix $\mathbf{C}^{*}$

4. Perform another $\mathrm{CA}$ on the resultant matrix $\mathbf{C}^{*}$ with modified block diagonal.

5. Repeat steps 3 and 4, that is substituting the diagonal blocks from the reconstructed values in the new solution, performing again the CA to obtain another solution, and so on, until the process converges. Convergence can be measured by the maximum absolute difference between the values substituted into the diagonal blocks at the present iteration and their corresponding values substituted during the previous iteration.

Figure 4 shows the results of a two-dimensional JCA applied to the Burt matrix of Table 3 , using a stopping criterion of $\varepsilon=0.0001$, where we have again left the scale exactly as in Figure 3. Comparing these two figures we can see the high degree of similarity in the pattern of the response categories, but mainly a change in the scale, with the JCA map being reduced in scale on both axes, but especially on the second. Most of the properties of simple CA carry over to JCA, most importantly the reconstruction of profiles with respect to biplot axes (Greenacre, 1993a, chapter 16).

Compared to regular CA and MCA there are two aspects to remember in order to compute the percentage of inertia explained by the map. First, the percentage has to be calculated for the two dimensions of the solution together, not separately, since the dimensions in JCA are not nested. Second, in the final solution (the CA of the modified Burt matrix at the final iteration) the usual way of calculating the proportion of explained inertia, involves the ratio between the sum of the first two principal inertias and the total inertia, but both the numerator and denominator of this sum include an amount due to the modified diagonal blocks, which are fitted exactly by the solution. This amount, which can be calculated in various ways (for example, by direct calculation on the modified blocks), 
needs to be discounted from both the numerator and denominator to obtain the percentage of (off-diagonal) inertia explained. In this particular example, the first two principal inertias of the modified Burt matrix are 0.09909 and 0.06503 respectively, and the total inertia including the part on the modified diagonal blocks is 0.18242 . The part on the modified diagonal blocks can be computed to be equal to 0.05474 , so that after discounting this amount from both numerator and denominator, we obtain the proportion of inertia explained by the two-dimensional JCA solution as

$$
\frac{0.09909+0.06503-0.05474}{0.18242-0.05474}=\frac{0.10938}{0.12768}=0.8567
$$

Hence $85.7 \%$ of the (off-diagonal) inertia is explained by the JCA solution, much higher than the $35.1 \%$ explained in the MCA map based on the Burt matrix. The value of $85.7 \%$ appropriately measures the success of approximating the off-diagonal blocks relative to the total inertia of these blocks only, unaffected by the diagonal blocks. This would be the quality of the map considered as a MCA biplot as well: that is, express all six off-diagonal blocks as profiles (rows or columns profiles, in upper or lower triangle of the Burt matrix), then the quality of reconstructing these profiles as described in Section 3.2 would be $85.7 \%$, and the error of reconstruction, or residual, would be $14.3 \%$.

\subsection{Adjustment of the inertias in MCA}

Since main difference between MCA and JCA in Figures 3 and 4 is a change in scale, it is possible to remedy partially the percentage of inertia problem in a regular MCA by a compromise between the MCA solution and the JCA objective, using simple scale readjustments of the MCA solution. In this approach the total inertia is measured (as in JCA) by the average inertia of all off-diagonal blocks of $\mathbf{C}$, calculated either directly from the tables themselves or by adjusting the total inertia of $\mathbf{C}$ by removing the fixed contributions of the diagonal blocks as follows:

$$
\text { average off-diagonal inertia }=\frac{Q}{Q-1}\left(\operatorname{inertia}(\mathbf{C})-\frac{J-Q}{Q^{2}}\right)
$$

Parts of inertia are then calculated from the principal inertias $\lambda_{s}{ }^{2}$ of $\mathbf{C}$ (or from the principal inertias $\lambda_{s}$ of $\mathbf{Z}$ ) as follows: for each $\lambda_{s} \geq 1 / Q$, calculate the adjusted inertias: 


$$
\lambda_{s}^{\mathrm{adj}}=\left(\frac{Q}{Q-1}\right)^{2}\left(\lambda_{s}-\frac{1}{Q}\right)^{2}
$$

and then express these as percentages of (12). Although these percentages underestimate those of a JCA, they dramatically improve the results of a MCA and are recommended in all applications of MCA. A further property of the adjusted principal inertias $\lambda_{s}^{\mathrm{adj}}$ is that they are identical to the principal inertias $\sigma_{s}^{2}$ of simple CA in the case of two categorical variables, where $Q=2: \lambda_{s}^{\mathrm{adj}}=4\left(\lambda_{s}-1 / 2\right)^{2}$, since we have shown earlier in Section 2.2 the relationship $\lambda_{s}=1 / 2\left(1+\sigma_{s}\right)$.

In our example the total inertia of $\mathbf{C}$ is equal to 1.1138 and the first seven principal inertias are such that $\lambda_{s} \geq 1 / Q$, that is $\lambda_{s}^{2} \geq 1 / Q^{2}=1 / 16$. The average off-diagonal inertia is equal to 0.17024 , as shown in Table 6 along with the different possibilities for inertias and percentages of inertia. Thus what appears to be a percentage explained in two dimensions of $22.2 \%(=11.4+10.8)$ in the analysis of the indicator matrix $\mathbf{Z}$, or $35.1 \%(=18.6+16.5)$ in the analysis of the Burt matrix $\mathbf{C}$, is shown to have a lower bound of $79.1 \%(=44.9+34.2)$ when the principal inertias are adjusted. Compared to the adjusted MCA solution, the JCA solution for this example (Figure 4) gives an additional benefit of 6.4 percentage points in the explained inertia, with a percentage explained of $85.7 \%$. We stress again that in JCA the solutions are not nested, so the percentages are reported for the whole solution (in this case, a two-dimensional one), not for individual dimensions.

We propose the adjusted solution as the one to be routinely reported: not only does it considerably improve the measure of fit, but it also removes the inconsistency about which of the two matrices to analyse, indicator or Burt. The adjusted solution is given in Figure 5, and has the same standard coordinates as Figure 3 but uses the adjusted principal inertias to calculate the principal coordinates, leading to the improved quality of display. Again we have left the scale identical to Figures 3 and 4 for purposes of comparison.

Benzécri (1979) has proposed the same adjusted inertias (14), but expresses them as percentages of their own sum over the dimensions $s$ for which $\lambda_{s} \geq 1 / Q$. This approach goes to the opposite extreme of giving an overly optimistic expression of explained inertia, since it explains a $100 \%$ in the space of the dimensions for which $\lambda_{s} \geq 1 / Q$ (there are six 
dimensions in our example - see Table 6) when in fact the data are not exactly reconstructed in the map.

\section{$4 \quad$ Supplementary points}

Up to now we have described three different ways to perform MCA:

Variant 1. CA of the indicator matrix

Variant 2. CA of the Burt matrix

Variant 3. An adjusted version of variants 1 or 2 that unifies and rectifies the scaling issue, giving a single solution irrespective of which matrix is considered, with highly improved measures of explained inertia

In all these variations, the standard coordinates of category points remain the same, only the principal inertias change. Furthermore, we have introduced an alternative method, JCA, which has a different solution from the above variants and which is analogous to leastsquares factor analysis in that it concentrates on between-variable associations only. In all of these cases it is possible to display supplementary points in the usual way to enrich the interpretation (see Le Roux and Rouanet, 2004, where this aspect is discussed at length in the context of MCA). Here we define a way of displaying supplementary points that does not depend on the variant of the method used. In our illustration, we will consider three supplementary demographic variables: gender, age and education - full category descriptions are given in Section 1.

To motivate our approach we consider the case of the indicator matrix, where supplementary categories can be thought of as either row or column points. For example, male and female categories can be added as two supplementary column dummy variables, or as two supplementary rows containing the frequencies for males and for females across the response categories. These two alternatives are equivalent up to scale factors, as we now explain. To position a supplementary column category, using the socalled transition, or barycentric, relationship between rows and columns (see, for example, Greenacre, 1984), we have to consider all the respondent points (rows) in standard coordinate positions in the map. Then any column category, active or supplementary, is situated (in principal 
coordinates) at the average of the respondents who fall into that category. Alternatively, to position a supplementary row, for example "male", we have to consider all the active column categories in standard coordinate positions, then the "male" row point will be at the weighted average of column points, using the profile of "male" across the active columns. Remember that the "male" frequency profile sums to 1 across the $Q$ questions, so its position is an average of averages: for each question the group "males" has an average position according to male frequencies across the categories of that particular question, and the final position of "male" is a simple average of these averages. We can show that the position of a supplementary point as a row is the same as the supplementary column dummy but shrunk on each dimension by the corresponding singular value, that is by the same scale factor that links principal to standard coordinates on each dimension (see Greenacre, 1984: Chapter 5.1 for a proof of this result). Thus a simple way to unify the representation of supplementary points in all situations would be to think of supplementary categories always as the averages of the principal coordinate positions of respondents, in which case both approaches will give exactly the same results.

Our proposal is thus the following: using the principal coordinates of respondent points, calculate average positions for supplementary categories, for example the average position for male points, female points, etc. Since it is only for the indicator matrix (variant 1 listed above) that we (automatically) have respondent points in the CA, we need to make precise what we mean by respondent points in the case of the Burt matrix and the adjusted analysis (variants 2 and 3 above, respectively). In these last cases, respondent points are displayed, at least theoretically, as supplementary points, that is as averages of the column categories, in standard coordinates, according to their respective response patterns. Since in all three variants of MCA these standard coordinates are identical, respondent points will have exactly the same positions in all three cases. Thus when we average their positions according to a supplementary variable, showing for example average male and average female points, the results will also be identical. But notice that to obtain these average positions we do not actually have to calculate all the original respondent points: the calculations can be made much more efficiently, thanks to transition relationships, by simply adding cross-tabulations as supplementary rows or columns. The following summarizes the calculations in each case, assuming that a supplementary variable is coded 
in indicator form as $\mathbf{Z}_{s}$, so that $\mathbf{Z}_{s}^{\top} \mathbf{Z}$ denotes the concatenated set of cross-tabulations of the supplementary variable with the $Q$ active variables:

1. in the case of the indicator matrix $\mathbf{Z}$, we would already have the principal coordinates of the responde nts, so we can either make the calculation of averages directly or add as supplementary row points the cross-tabulations $\mathbf{Z}_{s}^{\top} \mathbf{Z}$ of the supplementary variable with the active variables;

2. in the case of the Burt matrix $\mathbf{C}$, we do not need to calculate the positions of respondents (if required for other reasons, this would be done by adding $\mathbf{Z}$ as supplementary rows to the Burt matrix $\mathbf{C}$ ) - we can simply add the cross-tabulations $\mathbf{Z}_{s}^{\top} \mathbf{Z}$ as supplementary rows (or $\mathbf{Z}^{\top} \mathbf{Z}_{s}$ as supplementary columns), which leads to the same positions for the supplementary categories as in variant 1 ;

3. in the case of the adjusted analysis, we do as in variant 2 , since it is only $a$ posteriori that we adjust the eigenvalues, and this adjustment only affects the positions of the principal coordinates of the active category points, not the supplementary categories which - we repeat - are defined as averages of the respondent points;

4. in the case of JCA, it is again a simple addition of supplementary rows or columns, as in variants 2 and 3, to the modified Burt matrix $\mathbf{C}^{*}$ at the final iteration of the algorithm.

Table 7 shows the cross-tabulations $\mathbf{Z}_{s}^{\top} \mathbf{Z}$ and Figure 6 shows the positions of the supplementary points in the adjusted MCA (variant 3, that is the supplementary points are superimposed on Figure 5). Here we can see that the age groups and education groups show a horizontal trend, with younger respondents on the right moving over to older respondents on the left, and lower education groups on the left moving over to higher education groups on the right. In addition, we can see that the three upper education groups (from secondary education completed upwards) separate out at the right towards the strong disagreement poles of the questions, indicating they are particularly strongly in favour of science. We also find the average male point on the right hand side and average female point on the left hand side. Remember that these supplementary variables have been added separately to the map and not in combination - that is, the fact that male is on the right and 
higher education groups on the right does not imply that it is only higher-educated males that are more favourable towards science. To see the positions of higher-educated females, for example, interactive coding of the demographic variables would have to be performed: for example, coding the six education groups for males and for females separately, giving 12 combinations of gender and education, each represented as a separate supplementary point.

\section{$5 \quad$ Discussion and conclusions}

We have shown that extending CA of two variables to the case of several variables is not a simple issue, especially in the geometric case. Simple CA is typically applied to situations where two different types of variables are cross-tabulated, for example country of residence by responses to a survey question. MCA is applied to one set of variables, preferably all with the same response scales, which revolve around a particular issue, and where we are interested in the association patterns amongst the variables. Putting this in another way, in simple CA we are interested in associations between two variables or between two sets of variables, while in MCA we are interested in associations within a set of variables. This is an important difference between the two methods that permeates both the data analysis and interpretation of results.

Although the geometric concepts of simple CA do not carry over easily to the multiple-variable case, adjustment of the principal inertias and alternative methods such as JCA partially rectify the situation. Since MCA has attractive properties of optimality of scale values thanks to achieving maximum intercorrelation and thus maximum reliability (in terms of Cronbach's alpha), the compromise offered by the adjusted MCA solution is the most sensible one and the one that we recommend. The adjustment, described in Section 3.4, is easy to calculate and simply changes the scale on each dimension of the map to best approximate the two-tables of association between pairs of variables, leaving everything else in the solution intact. Thanks to this adjustment we obtain estimates of the explained inertias that are much closer to the true values than the pessimistic values obtained in MCA - for this reason we propose the adjusted MCA solution as the one to be used as a matter of course in all (graphical) MCA applications. The adjusted solution also 
has the nice property that it is identical to simple CA of a cross-tabulation in the case of two variables. JCA also perfectly reproduces simple CA in the two variable case, since is is also focused exclusively on the on the single off-diagonal cross-tabulation. JCA has the additional advantage in the multivariable case of optimizing the fit to all the off-diagonal cross-tabulations of interest.

Categorical supplementary points can be added to any of the variants of MCA, as well as JCA, as averages of respondents that fall into the corresponding categories. This amounts to simply adding the cross-tabulations of the supplementary variables with the active variables as supplementary rows or columns. The JCA and adjusted MCA solutions have the advantage that the active points are reduced in scale compared to the solutions for the indicator and Burt matrices, thus leading to a greater relative dispersion of the supplementary points in the joint map of the active and supplementary points.

\section{Software note}

The analyses of this chapter were performed using XLSTAT (www.xlstat.com) written by Thierry Fahmy, and cross-checked using the R functions for CA, MCA and JCA written by Oleg Nenadic. Both implementations include the adjustment of inertias described in this paper.

\section{References}

Boik, R.J. (1996). An efficient algorithm for joint correspondence analysis. Psychometrika, 61, 255-269.

Gifi, A. (1990). Nonlinear Multivariate Analysis. Chichester, UK: Wiley.

Gower, J.C. (1993). The construction of neighbour-regions in two dimensions for prediction with multi-level categorical variables. In O. Opitz, B. Lausen and R. Klar (eds), Information and Classification: Concepts-Methods-Applications, pp. 174-189. Berlin: Springer. 
Greenacre, M.J. and Harding, S.A. (1998). Prediction regions for categorical variables. In J. Blasius and M.Greenacre (eds), Visualization of Categorical Data, pp. 405-419. San Diego: Academic Press.

Greenacre, M.J. (1984). Theory and Applications of Correspondence Analysis. London: Academic Press.

Greenacre, M.J. (1988). Correspondence analysis of multivariate categorical data by weighted least squares. Biometrika, 75, 457-467.

Greenacre, M.J. (1989). The Carroll-Green-Schaffer in correspondence analysis: a theoretical and empirical appraisal. Journal of Marketing Research, 26, 358-365.

Greenacre, M.J. (1993a). Correspondence Analysis in Practice. London: Academic Press.

Greenacre, M.J. (1993b). Biplots in correspondence analysis. Journal of Applied Statistics, 20, 251-269.

Greenacre, M.J. (1994). Multiple and joint correspondence analysis. In M.Greenacre and J. Blasius (eds), Correspondence Analysis in the Social Sciences, pp. 141-161. London: Academic Press.

Greenacre, M.J. (1998). Diagnostics for joint displays in correspondence analysis. In J. Blasius and M.Greenacre (eds), Visualization of Categorical Data, pp. 221-238. San Diego: Academic Press.

Greenacre, M.J. and Hastie, T.J. (1987). The geometric interpretation of correspondence analysis. Journal of the American Statistical Association, 82, 437-447.

Greenacre, M.J. and Pardo, R. (2005). Multiple correspondence analysis of a subset of response categories. Working report no. 881, Department of Economics and Business, Universitat Pompeu Fabra, Barcelona.

Nishisato, S.(1994). Elements of Dual Scaling: an Introduction to Practical Data Analysis. Hillsdale: Lawrence Erlbaum.

Tateneni, K. and Browne, M.W. (2000). A non-iterative method of joint correspondence analysis. Psychometrika, 65, 157-165. 


\section{Table 1}

Cross -tabulation of 871 West German respondents with respect to two questions on attitudes to science.

\begin{tabular}{|c|c|c|c|c|c|c|c|}
\hline \multirow[b]{3}{*}{ We believe } & & \multicolumn{5}{|c|}{ Overall, modern science does more harm than good } & \multirow[b]{2}{*}{ SUM } \\
\hline & \multicolumn{2}{|r|}{$\begin{array}{l}\text { B1-agree } \\
\text { strongly }\end{array}$} & B2-agree & $\begin{array}{l}\text { B3-neither / } \\
\text { nor }\end{array}$ & B4-disagree & $\begin{array}{c}\text { B5-disagree } \\
\text { strongly }\end{array}$ & \\
\hline & A1-aqree strongly & 27 & 28 & 30 & 22 & 12 & 119 \\
\hline too often in & A2-agree & 38 & 74 & 84 & 96 & 30 & 322 \\
\hline science, not & A3-neither/nor & 3 & 48 & 63 & 73 & 17 & 204 \\
\hline enough in & A4-disagree & 3 & 21 & 23 & 79 & 52 & 178 \\
\hline feelings \& faith & A5-disagree strongly & 0 & 3 & 5 & 11 & 29 & 48 \\
\hline & SUM & 71 & 174 & 205 & 281 & 140 & 871 \\
\hline
\end{tabular}


Table 2

Data on attitudes to science and the environment, showing the complete Burt matrix of all pairwise crosstables of the four variables. Table 1 is the $\mathrm{A} \times \mathrm{B}$ block of this matrix.

\begin{tabular}{|c|c|c|c|c|c|c|c|c|c|c|c|c|c|c|c|c|c|c|c|c|}
\hline & $\mathrm{A} 1$ & A2 & A3 & A4 & A5 & B1 & B2 & B3 & B4 & B5 & $\mathrm{C} 1$ & $\mathrm{C} 2$ & C3 & $\mathrm{C} 4$ & C5 & D1 & D2 & D3 & D 4 & D5 \\
\hline $\mathrm{A} 1$ & 119 & 0 & 0 & 0 & 0 & 27 & 28 & 30 & 22 & 12 & 49 & 40 & 18 & 7 & 5 & 15 & 25 & 17 & 34 & 28 \\
\hline A2 & 0 & 322 & 0 & 0 & 0 & 38 & 74 & 84 & 96 & 30 & 67 & 142 & 60 & 41 & 12 & 22 & 102 & 76 & 68 & 54 \\
\hline A 3 & 0 & 0 & 204 & 0 & 0 & 3 & 48 & 63 & 73 & 17 & 18 & 75 & 70 & 34 & 7 & 10 & 44 & 68 & 58 & 24 \\
\hline A 4 & 0 & 0 & 0 & 178 & 0 & 3 & 21 & 23 & 79 & 52 & 16 & 50 & 40 & 56 & 16 & 9 & 52 & 28 & 54 & 35 \\
\hline A5 & 0 & 0 & 0 & 0 & 48 & 0 & 3 & 5 & 11 & 29 & 2 & 9 & 9 & 16 & 12 & 4 & 9 & 13 & 12 & 10 \\
\hline B1 & 27 & 38 & 3 & 3 & 0 & 71 & 0 & 0 & 0 & 0 & 43 & 19 & 4 & 3 & 2 & 9 & 17 & 10 & 10 & 25 \\
\hline B2 & 28 & 74 & 48 & 21 & 3 & 0 & 174 & 0 & 0 & 0 & 36 & 88 & 34 & 15 & 1 & 16 & 51 & 42 & 45 & 20 \\
\hline B3 & 30 & 84 & 63 & 23 & 5 & 0 & 0 & 205 & 0 & 0 & 37 & 90 & 57 & 19 & 2 & 10 & 53 & 63 & 51 & 28 \\
\hline B4 & 22 & 96 & 73 & 79 & 11 & 0 & 0 & 0 & 281 & 0 & 27 & 88 & 75 & 74 & 17 & 6 & 66 & 70 & 92 & 47 \\
\hline B5 & 12 & 30 & 17 & 52 & 29 & 0 & 0 & 0 & 0 & 140 & 9 & 31 & 27 & 43 & 30 & 19 & 45 & 17 & 28 & 31 \\
\hline $\mathrm{C} 1$ & 49 & 67 & 18 & 16 & 2 & 43 & 36 & 37 & 27 & 9 & 152 & 0 & 0 & 0 & 0 & 25 & 24 & 15 & 38 & 50 \\
\hline $\mathrm{C} 2$ & 40 & 142 & 75 & 50 & 9 & 19 & 88 & 90 & 88 & 31 & 0 & 316 & 0 & 0 & 0 & 15 & 97 & 67 & 89 & 48 \\
\hline C3 & 18 & 60 & 70 & 40 & 9 & 4 & 34 & 57 & 75 & 27 & 0 & 0 & 197 & 0 & 0 & 5 & 51 & 83 & 41 & 17 \\
\hline $\mathrm{C} 4$ & 7 & 41 & 34 & 56 & 16 & 3 & 15 & 19 & 74 & 43 & 0 & 0 & 0 & 154 & 0 & 6 & 44 & 30 & 51 & 23 \\
\hline C5 & 5 & 12 & 7 & 16 & 12 & 2 & 1 & 2 & 17 & 30 & 0 & 0 & 0 & 0 & 52 & 9 & 16 & 7 & 7 & 13 \\
\hline D1 & 15 & 22 & 10 & 9 & 4 & 9 & 16 & 10 & 6 & 19 & 25 & 15 & 5 & 6 & 9 & 60 & 0 & 0 & 0 & 0 \\
\hline D2 & 25 & 102 & 44 & 52 & 9 & 17 & 51 & 53 & 66 & 45 & 24 & 97 & 51 & 44 & 16 & 0 & 232 & 0 & 0 & 0 \\
\hline D3 & 17 & 76 & 68 & 28 & 13 & 10 & 42 & 63 & 70 & 17 & 15 & 67 & 83 & 30 & 7 & 0 & 0 & 202 & 0 & 0 \\
\hline D 4 & 34 & 68 & 58 & 54 & 12 & 10 & 45 & 51 & 92 & 28 & 38 & 89 & 41 & 51 & 7 & 0 & 0 & 0 & 226 & 0 \\
\hline D5 & 28 & 54 & 24 & 35 & 10 & 25 & 20 & 28 & 47 & 31 & 50 & 48 & 17 & 23 & 13 & 0 & 0 & 0 & 0 & 151 \\
\hline
\end{tabular}




\section{Table 3}

Results of CA of $871 \times 20$ indicator matrix $\mathbf{Z}$, or equivalently of Burt matrix $\mathbf{C}$ in Table 2, showing standard coordinates (scale values) for the four variables on the first two dimensions of the solution (F1 and F2); "sq.corr." is the squared correlation of the quantified variable with the total score, and "rho" is the corresponding singular value of $\mathbf{C}$, i.e. the squared singular value (or principal inertia) of $\mathbf{Z}$, which is the arithmetic average of the four corresponding squared correlations. Cronbach's alpha is the measure of reliability discussed in Section 2.2.

\begin{tabular}{lrr} 
& $\mathrm{F} 1$ & $\mathrm{~F} 2$ \\
\hline A1 & -1.837 & 0.727 \\
A2 & -0.546 & -0.284 \\
A3 & 0.447 & -1.199 \\
A4 & 1.166 & 0.737 \\
A5 & 1.995 & 2.470 \\
\hline sq.corr. & 0.510 & 0.382 \\
\hline B1 & -2.924 & 1.370 \\
B2 & -0.642 & -0.667 \\
B3 & -0.346 & -0.964 \\
B4 & 0.714 & -0.280 \\
B5 & 1.354 & 2.108 \\
\hline sq.corr. & 0.579 & 0.517 \\
\hline C1 & -2.158 & 0.909 \\
C2 & -0.247 & -0.592 \\
C3 & 0.619 & -1.044 \\
C4 & 1.349 & 0.635 \\
C5 & 1.468 & 3.017 \\
\hline sq.corr. & 0.627 & 0.488 \\
\hline D1 & -1.204 & 1.822 \\
D2 & 0.221 & -0.007 \\
D3 & 0.385 & -1.159 \\
D4 & 0.222 & -0.211 \\
D5 & -0.708 & 1.152 \\
\hline sq.corr. & 0.113 & 0.337 \\
\hline & & \\
rho & $\mathbf{0 . 4 5 7}$ & $\mathbf{0 . 4 3 1}$ \\
\hline Cronbach's alpha & $\mathbf{0 . 6 0 5}$ & $\mathbf{0 . 5 6 0}$
\end{tabular}




\section{Table 4}

In the upper triangle, in italics: the squared correlations between the four variables A, B, C and $\mathrm{D}$, quantified by their scale values on the first dimension, as well as their squared correlations with the total score (cf. column F1 of Table 3). In the diagonal and lower triangle: the variances and covariances respectively, with the average covariance of each variable with itself and the others in the last line in boldface (e.g., $0.5793=$ $(0.4440+1.2666+0.5697+0.0369) / 4)$. Notice that these average covariances are identical to the squared correlations with the total. Hence, the variance of the average score (the quantity maximized by MCA, underlined) is both the average of the four squared correlations of the question scores with the total score, and the average of the four average covariances, in other words the average of the full $4 \times 4$ variance-covariance matrix. Notice further that the sum of the variances of the four variables $1.1151+1.2666+1.3716+0.2467=$ 4 , which is the identification condition on the scale values. (To calculate variances and covariances, divide by $n=871$, not $n-1$.)

$\begin{array}{cccccc} & \text { A } & \text { B } & \text { C } & \text { D } & \begin{array}{c}\text { squared } \\ \text { correlation } \\ \text { with total }\end{array} \\ \text { A } & 1.1151 & 0.1396 & 0.1270 & 0.0059 & \mathbf{0 . 5 1 0 0} \\ \text { B } & 0.4440 & 1.2666 & 0.1868 & 0.0059 & \mathbf{0 . 5 7 9 3} \\ \text { C } & 0.4406 & 0.5697 & 1.3716 & 0.0480 & \mathbf{0 . 6 2 7 3} \\ \text { D } & 0.0403 & 0.0369 & 0.1274 & 0.2467 & \mathbf{0 . 1 1 2 9} \\ \text { average } & \mathbf{0 . 5 1 0 0} & \mathbf{0 . 5 7 9 3} & \mathbf{0 . 6 2 7 3} & \mathbf{0 . 1 1 2 9} & \mathbf{0 . 4 5 7 4}\end{array}$


Table 5

(a) Row profiles of Table 1, including average row profile; (b) Approximate row profiles estimated from biplot of Figure 2 (the average profile is always represented exactly by the origin of the map). The difference between the two tables is the error of biplot approximation, measured as $100-95.6 \%=4.4 \%$ of the total inertia of the table.

(a) Original profiles

\begin{tabular}{llllll|c}
\hline & B1 & B2 & B3 & B4 & B5 & Sum \\
\hline A1 & 0.227 & 0.235 & 0.252 & 0.185 & 0.101 & 1 \\
A2 & 0.118 & 0.230 & 0.261 & 0.298 & 0.093 & 1 \\
A3 & 0.015 & 0.235 & 0.309 & 0.358 & 0.083 & 1 \\
A4 & 0.017 & 0.118 & 0.129 & 0.444 & 0.292 & 1 \\
A5 & 0.000 & 0.063 & 0.104 & 0.229 & 0.604 & 1 \\
\hline Average & 0.075 & 0.176 & 0.211 & 0.303 & 0.235 & 1 \\
\hline
\end{tabular}

(b) Estimated profiles

\begin{tabular}{cccccc|c}
\hline & B1 & B2 & B3 & B4 & B5 & Sum \\
\hline A1 & 0.226 & 0.239 & 0.253 & 0.181 & 0.102 & 1 \\
A2 & 0.117 & 0.229 & 0.265 & 0.294 & 0.094 & 1 \\
A3 & 0.024 & 0.226 & 0.283 & 0.393 & 0.074 & 1 \\
A4 & 0.002 & 0.135 & 0.169 & 0.387 & 0.307 & 1 \\
A5 & 0.026 & 0.034 & 0.034 & 0.329 & 0.578 & 1 \\
\hline Average & 0.075 & 0.176 & 0.211 & 0.303 & 0.235 & 1 \\
\hline
\end{tabular}




\section{Table 6}

Eigenvalues (principal inertias) of Burt matrix, their percentages of inertia, the adjusted inertias and their lower bound estimates of the percentages of explained inertia for the offdiagonal tables of the Burt matrix. The average off-diagonal inertia on which these latter percentages are based is equal to $\frac{4}{3}\left(1.12768-\frac{16}{16}\right)=0.17024$, where 1.12768 is the total inertia of the Burt matrix (that is the average of the inertias of all its submatrices, including those on the block diagonal). Notice that the sum of explain ed inertia over these six dimensions must be less than $100 \%$ in the case of the adjusted eigenvalues, since the percentages are lower bound estimates and, in any case, the six-dimensional MCA solution does not fully explain all pairwise cross-tabulations.

\begin{tabular}{ccccccc} 
Dimension & $\begin{array}{c}\text { Eigenvalue } \\
\text { of } \mathbf{Z}\end{array}$ & $\begin{array}{c}\text { Percentage } \\
\text { explained }\end{array}$ & $\begin{array}{c}\text { Eigenvalue } \\
\text { of } \mathbf{B}\end{array}$ & $\begin{array}{c}\text { Percentage } \\
\text { explained }\end{array}$ & $\begin{array}{c}\text { Adjusted } \\
\text { eigenvalue }\end{array}$ & $\begin{array}{c}\text { Percentage } \\
\text { explained }\end{array}$ \\
\hline 1 & 0.4574 & $11.4 \%$ & 0.2092 & $18.6 \%$ & 0.07646 & $44.9 \%$ \\
2 & 0.4310 & $10.8 \%$ & 0.1857 & $16.5 \%$ & 0.05822 & $34.2 \%$ \\
3 & 0.3219 & $8.0 \%$ & 0.1036 & $9.2 \%$ & 0.00920 & $5.4 \%$ \\
4 & 0.3065 & $7.7 \%$ & 0.0939 & $8.3 \%$ & 0.00567 & $3.3 \%$ \\
5 & 0.2757 & $6.9 \%$ & 0.0760 & $6.7 \%$ & 0.00117 & $0.7 \%$ \\
6 & 0.2519 & $6.3 \%$ & 0.0635 & $5.6 \%$ & 0.00001 & $0.0 \%$ \\
\hline
\end{tabular}




\section{Table 7}

Supplementary rows added to the indicator matrix $\mathbf{Z}$, Burt matrix $\mathbf{C}$ or modified Burt matrix $\mathbf{C}^{*}$ to represent the supplementary variables sex, age and education. These are the cross-tabulations of the three variables with the four active variables A, B, C and D. The supplementary categories are M: male, F: female, age 1: 16-24 years, age 2: 25-34 years, age 3: 35-44 years, age 4: 45-54 years, age 5: 55-64 years, age 6: 65 years and older, edu1: no or some primary education, edu2: primary education completed, edu 3 : some secondary education, edu 4 : secondary education completed, edu 5: some tertiary education, edu 6 : tertiary education completed.

\begin{tabular}{|c|c|c|c|c|c|c|c|c|c|c|c|c|c|c|c|c|c|c|c|c|}
\hline & $\mathrm{A} 1$ & A 2 & A3 & A4 & A5 & B1 & B2 & B3 & B4 & B5 & $\mathrm{C} 1$ & $\mathrm{C} 2$ & C3 & $\mathrm{C} 4$ & C5 & D1 & D2 & D3 & D 4 & D5 \\
\hline M & 43 & 144 & 120 & 92 & 28 & 25 & 75 & 104 & 146 & 77 & 58 & 157 & 105 & 82 & 25 & 25 & 136 & 92 & 101 & 73 \\
\hline F & 76 & 178 & 84 & 86 & 20 & 46 & 99 & 101 & 135 & 63 & 94 & 159 & 92 & 72 & 27 & 35 & 96 & 110 & 125 & 78 \\
\hline age1 & 9 & 34 & 17 & 25 & 6 & 5 & 21 & 16 & 33 & 16 & 13 & 32 & 22 & 19 & 5 & 7 & 26 & 19 & 30 & 9 \\
\hline age2 & 33 & 68 & 45 & 51 & 13 & 15 & 30 & 52 & 76 & 37 & 35 & 60 & 57 & 43 & 15 & 10 & 46 & 48 & 56 & 50 \\
\hline age 3 & 18 & 63 & 40 & 26 & 11 & 15 & 27 & 31 & 62 & 23 & 24 & 61 & 35 & 25 & 13 & 12 & 39 & 42 & 35 & 30 \\
\hline age 4 & 19 & 45 & 47 & 27 & 8 & 13 & 29 & 34 & 41 & 29 & 24 & 57 & 33 & 26 & 6 & 10 & 39 & 38 & 38 & 21 \\
\hline age 5 & 18 & 43 & 33 & 25 & 5 & 10 & 26 & 35 & 37 & 16 & 21 & 45 & 26 & 24 & 8 & 9 & 48 & 23 & 28 & 16 \\
\hline age 6 & 22 & 69 & 22 & 24 & 5 & 13 & 41 & 37 & 32 & 19 & 35 & 61 & 24 & 17 & 5 & 12 & 34 & 32 & 39 & 25 \\
\hline edul & 7 & 15 & 7 & 8 & 1 & 6 & 10 & 11 & 7 & 4 & 7 & 8 & 11 & 12 & 0 & 5 & 9 & 12 & 8 & 4 \\
\hline edu2 & 59 & 155 & 84 & 68 & 12 & 34 & 93 & 95 & 112 & 44 & 79 & 156 & 73 & 54 & 16 & 28 & 110 & 90 & 95 & 55 \\
\hline edu3 & 29 & 84 & 65 & 54 & 10 & 19 & 47 & 55 & 82 & 39 & 34 & 90 & 68 & 36 & 14 & 11 & 69 & 58 & 63 & 41 \\
\hline edu 4 & 11 & 27 & 18 & 26 & 12 & 6 & 12 & 18 & 37 & 21 & 18 & 24 & 18 & 28 & 6 & 7 & 13 & 23 & 29 & 22 \\
\hline edu 5 & 5 & 20 & 11 & 8 & 5 & 4 & 5 & 11 & 16 & 13 & 6 & 14 & 14 & 9 & 6 & 4 & 12 & 7 & 14 & 12 \\
\hline edu 6 & 8 & 21 & 19 & 14 & 8 & 2 & 7 & 15 & 27 & 19 & 8 & 24 & 13 & 15 & 10 & 5 & 19 & 12 & 17 & 17 \\
\hline
\end{tabular}




\section{Figure 1}

Symmetric CA map of Table 1. Percentage of inertia displayed in the map is $95.6 \%$.

Categories for questions A and B are from (1) agree strongly to (5) disagree strongly.

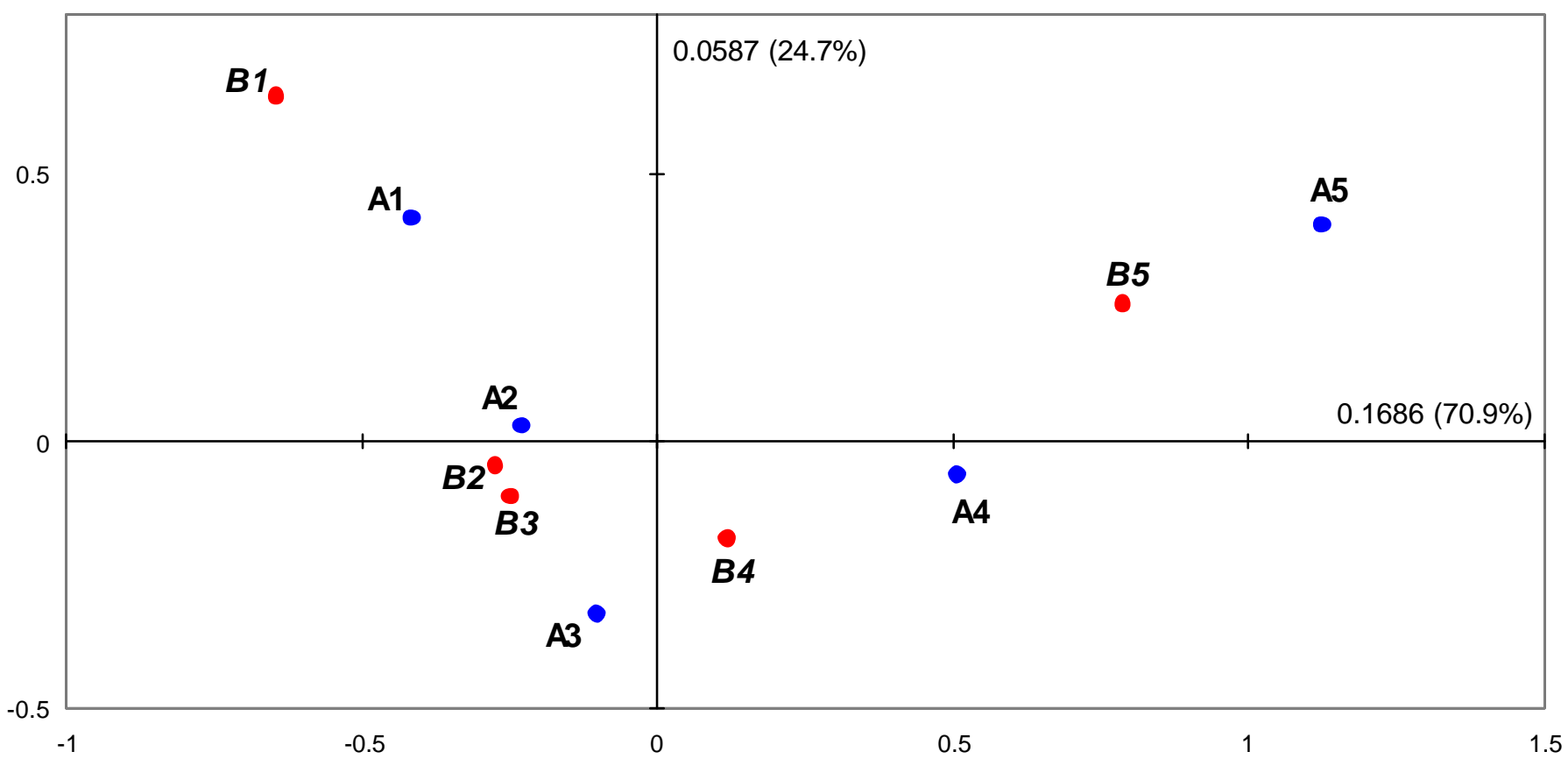




\section{Figure 2}

Asymmetric CA map of Table 1, showing biplot axes. The categories of the row points A1 to A5 can be projected onto each biplot axis to read off approximations of corresponding profile values. The accuracy of this approximation is as good as the percentage of inertia displayed, which is $95.6 \%$, hence it is excellent.

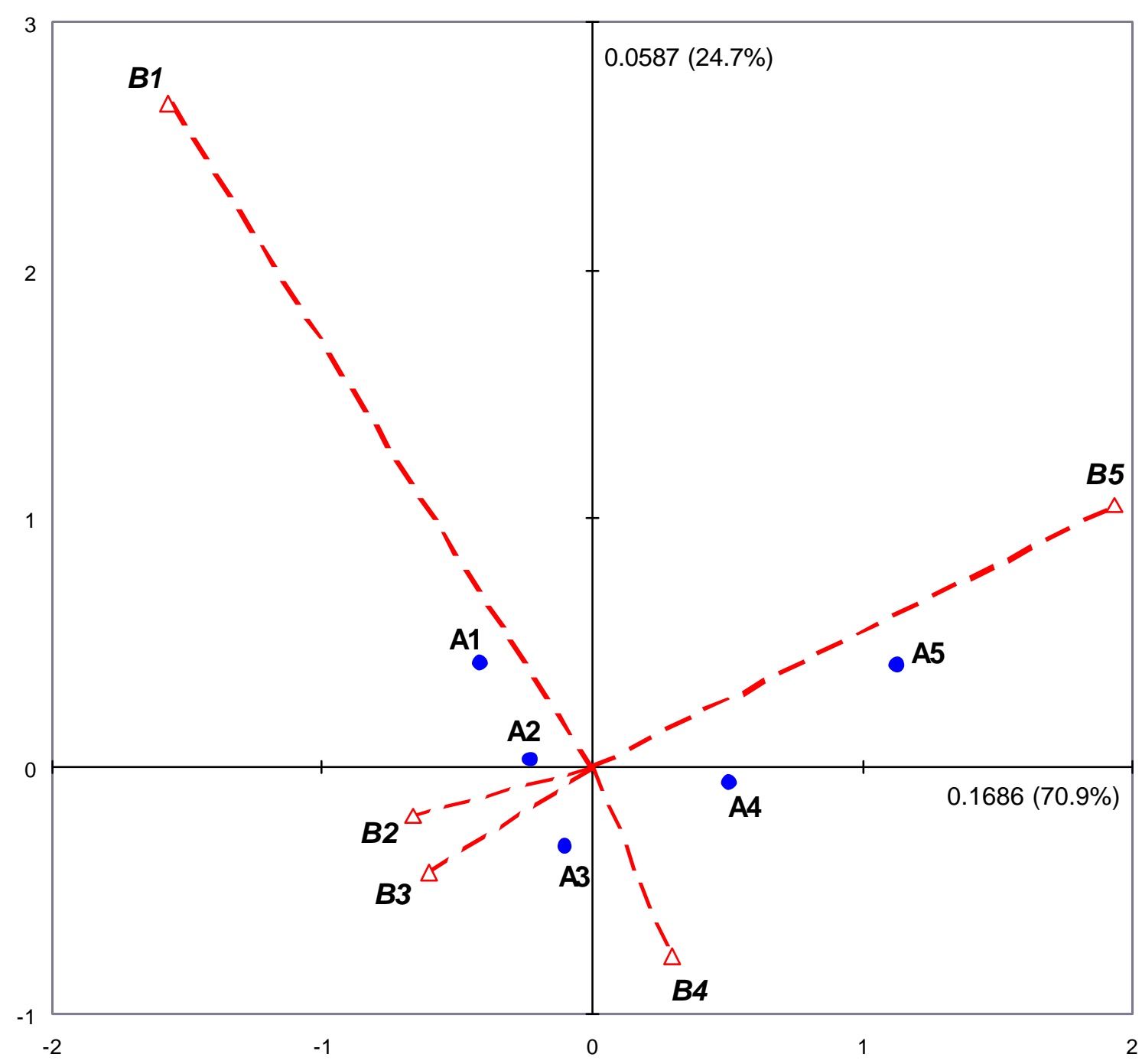


Figure 3

Multiple correspondence analysis map of the Burt matrix of Table 3. The percentage of explained inertia is $35.1 \%$.

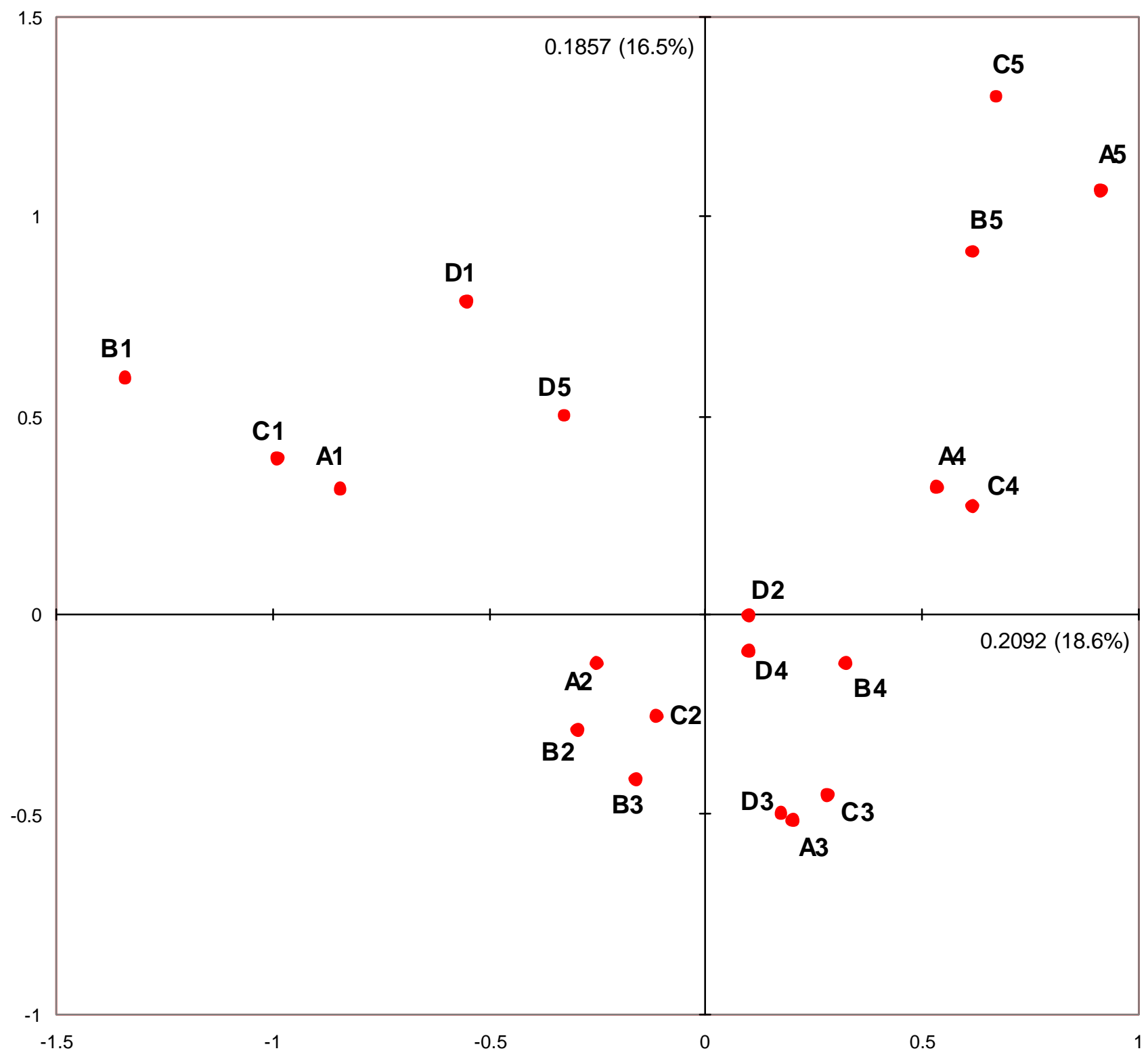




\section{Figure 4}

Joint correspondence analysis map of the Burt matrix of Table 3. The percentage of explained inertia is $85.7 \%$.

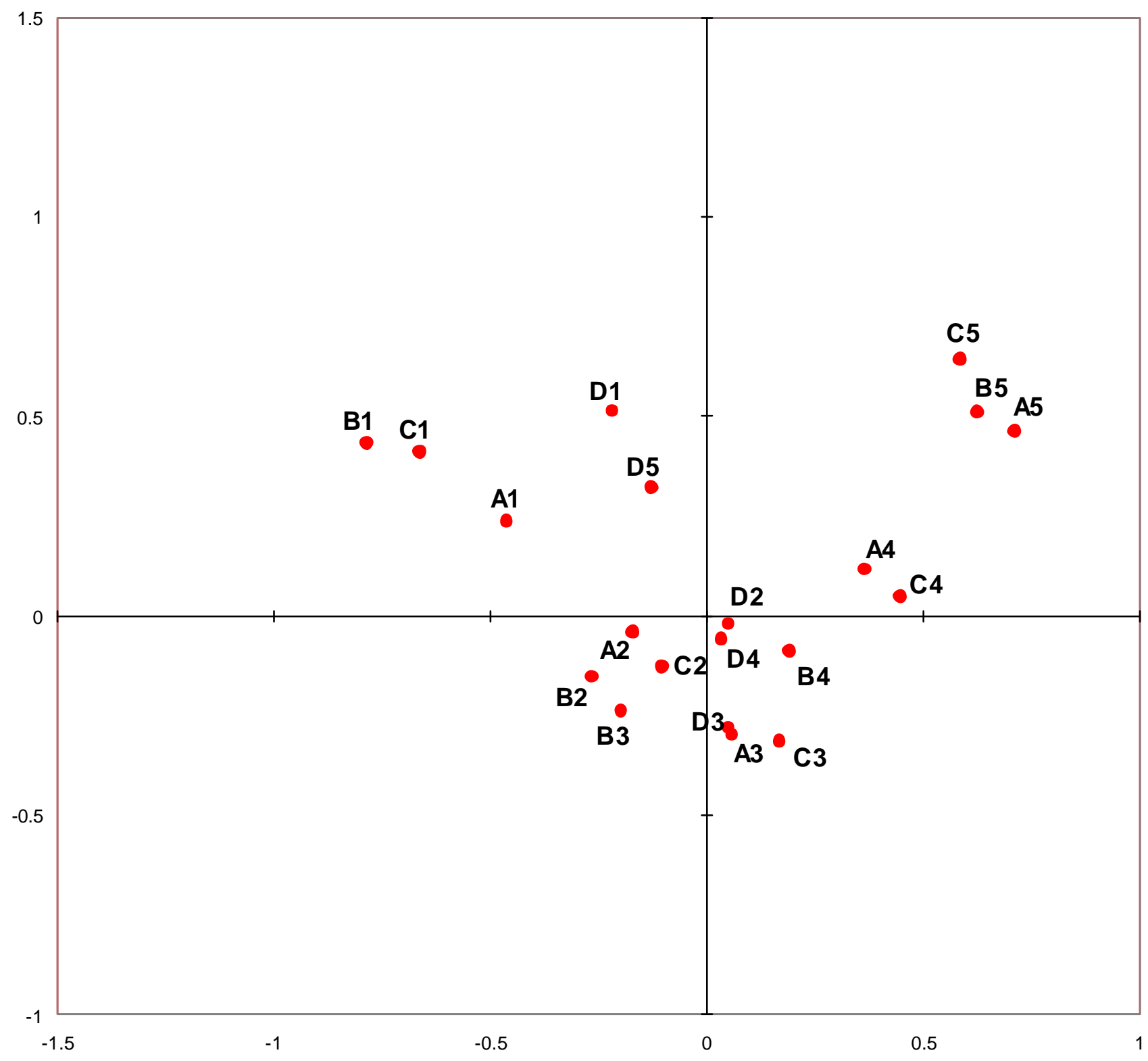




\section{Figure 5}

Multiple correspondence map of Table 3 with adjustment of the principal inertias (and thus the scale of the principal coordinates) along each dimension. The percentage of explained inertia is at least $79.1 \%$. Notice the change in scale compared to Figure 3.

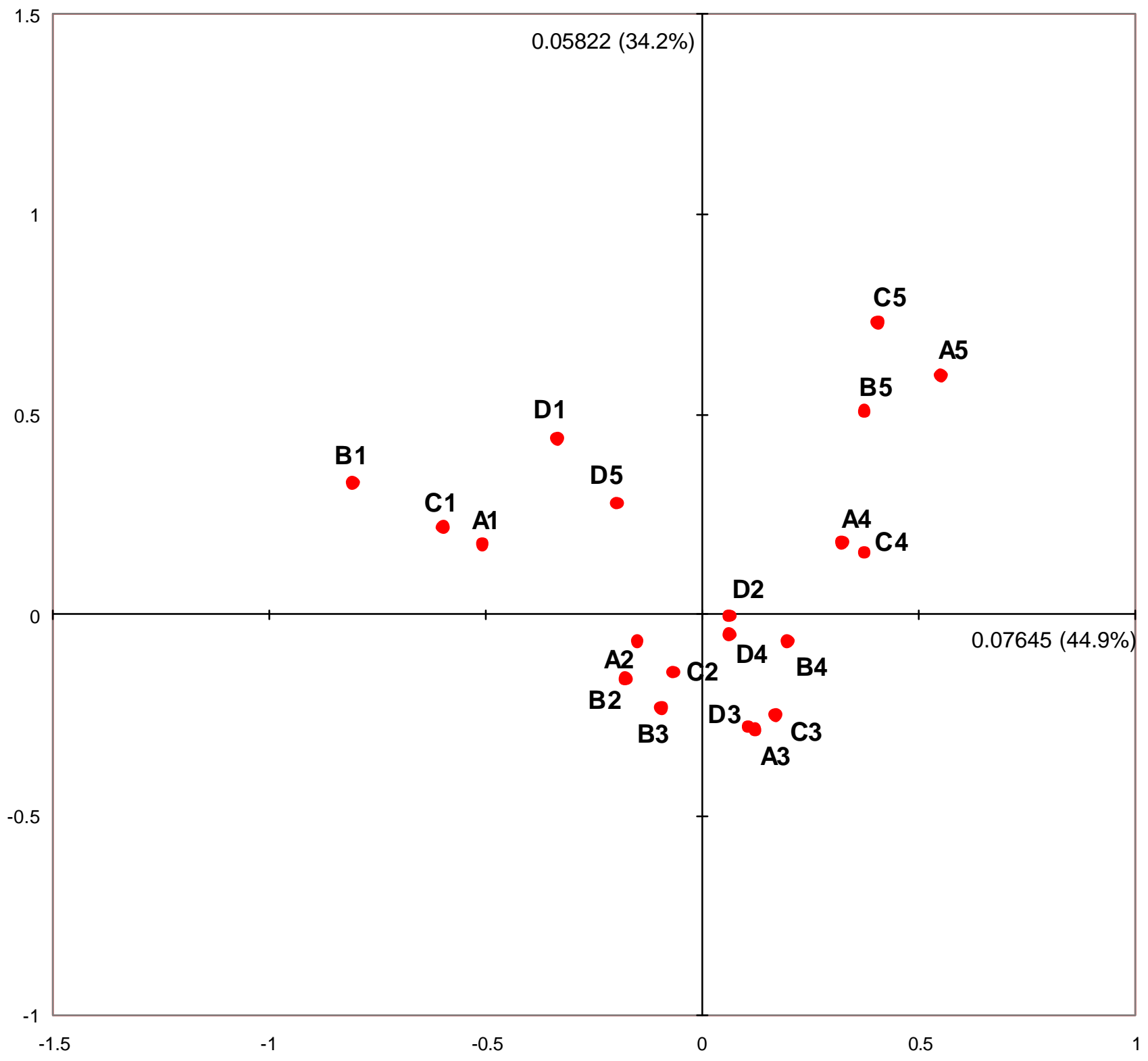




\section{Figure 6}

Adjusted MCA solution, showing positions of supple mentary categories of sex, age and education.

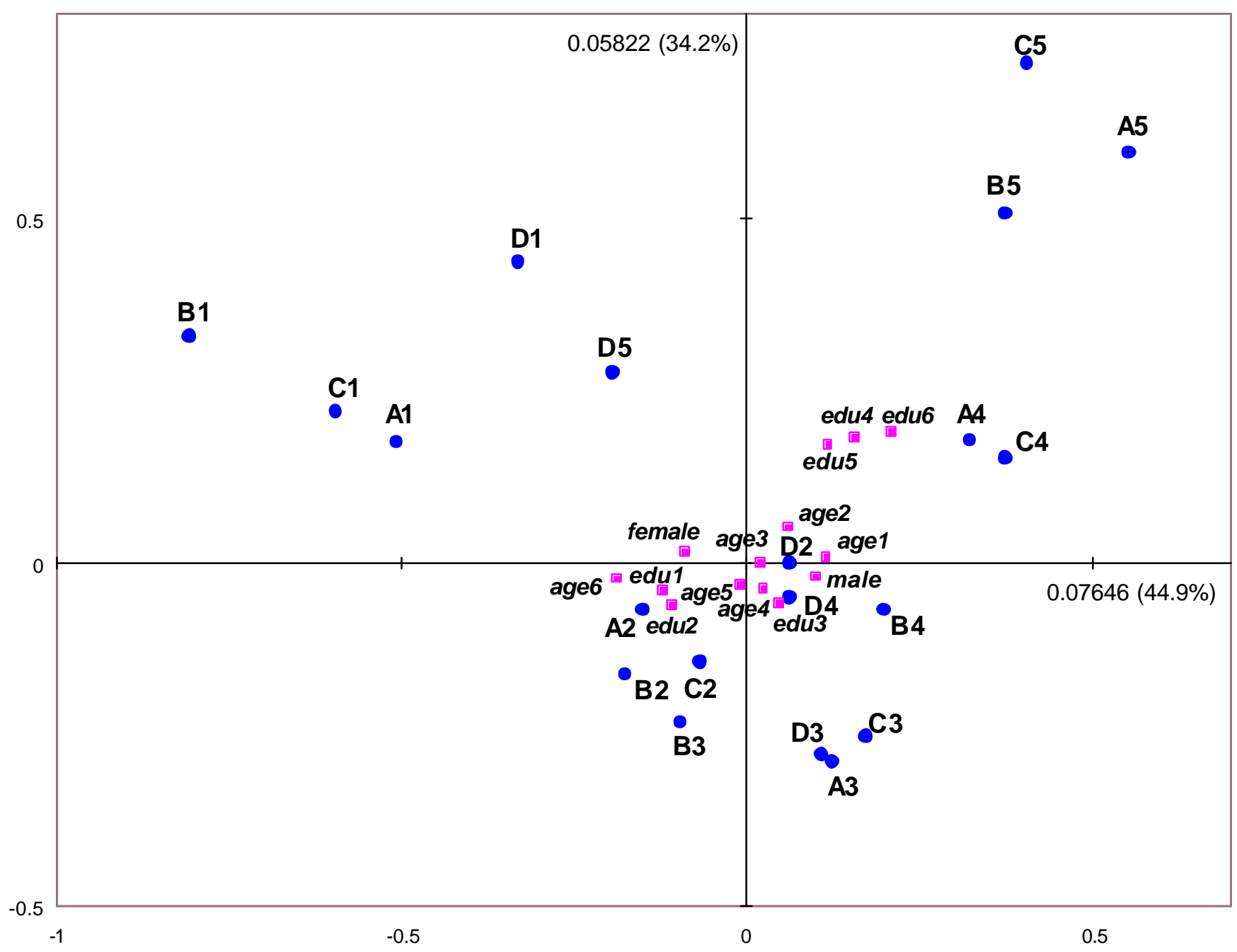

